\title{
New Modified Adomian Decomposition Recursion Schemes for Solving Certain Types of Nonlinear Fractional Two-Point Boundary Value Problems
}

\author{
Sekson Sirisubtawee and Supaporn Kaewta \\ Department of Mathematics, Faculty of Applied Science, King Mongkut's University of Technology North Bangkok, \\ Bangkok 10800, Thailand \\ Correspondence should be addressed to Sekson Sirisubtawee; sekson.s@sci.kmutnb.ac.th
}

Received 31 March 2017; Accepted 30 May 2017; Published 10 July 2017

Academic Editor: A. Zayed

Copyright (c) 2017 Sekson Sirisubtawee and Supaporn Kaewta. This is an open access article distributed under the Creative Commons Attribution License, which permits unrestricted use, distribution, and reproduction in any medium, provided the original work is properly cited.

\begin{abstract}
We apply new modified recursion schemes obtained by the Adomian decomposition method (ADM) to analytically solve specific types of two-point boundary value problems for nonlinear fractional order ordinary and partial differential equations. The new modified recursion schemes, which sometimes utilize the technique of Duan's convergence parameter, are derived using the DuanRach modified ADM. The Duan-Rach modified ADM employs all of the given boundary conditions to compute the remaining unknown constants of integration, which are then embedded in the integral solution form before constructing recursion schemes for the solution components. New modified recursion schemes obtained by the method are generated in order to analytically solve nonlinear fractional order boundary value problems with a variety of two-point boundary conditions such as Robin and separated boundary conditions. Some numerical examples of such problems are demonstrated graphically. In addition, the maximal errors $\left(\mathrm{ME}_{n}\right)$ or the error remainder functions $\left(\mathrm{ER}_{n}(x)\right)$ of each problem are calculated.
\end{abstract}

\section{Introduction}

During the last three decades, fractional order differential equations (FDEs) have played an important role in modelling many phenomena in engineering $[1,2]$, applied sciences [36], and biological systems $[7,8]$. This is because the behavior of most systems appear after effects or memory which can be explained better by using fractional order derivatives [5]. Several methods have been proposed to analytically and numerically solve nonlinear fractional order differential equations including initial value problems (IVPs) and boundary value problems (BVPs). These methods include the Adomian decomposition method (ADM) [9-14], the multistep generalized differential transform method (MSGDTM) [15], the Adams-Bashforth-Moulton type predictor-corrector scheme [16], and the Haar wavelet method [17].

The ADM has been extensively utilized to solve IVPs and BVPs for nonlinear ordinary or partial differential equations, integral equations, or integrodifferential equations since it can provide approximate analytic solutions without linearization, perturbation, discretization, guessing the initial term, or using Green's functions which are quite difficult to determine in most cases. The ADM has been used as a tool to investigate analytical and numerical solutions of real-world problems by Hashim et al. [18] and Sweilam and Khader [19]. Modifications of the ADM have been developed for different purposes for IVPs for both integer order and fractional order differential equations.

Several researchers proposed expressing the initial solution component $u_{0}$ as a series of orthogonal polynomials, such as Chebyshev polynomials [20], or Legendre polynomials [21, 22]. In 2013 Duan et al. [11] combined the ADM with convergence acceleration techniques such as diagonal Padé approximants and iterated Shanks transforms to solve nonlinear fractional ordinary differential equations. It was found that the modified techniques can efficiently extend the convergence region of the decomposition series solution. In 2014 Ramana and Prasad [23] modified the ADM to 
solve parabolic equations and the results obtained by their modified method converged very quickly and were more accurate than the standard ADM results.

There are now several different resolution techniques using the ADM for solving BVPs of nonlinear integer order differential equations. These techniques were developed by many authors as follows. Tatari and Dehghan [24] gave the solution of the general form of multipoint BVPs using the ADM. Al-Hayani [25] used the ADM with Green's function to solve sixth-order BVPs. Duan and Rach [26] proposed the Duan-Rach modified decomposition method for solving BVPs for higher order nonlinear differential equations. Duan et al. [27] developed the multistage ADM for solving BVPs for second-order nonlinear ordinary differential equations with Robin boundary conditions. In particular, modified approaches for solving nonlinear fractional order BVPs can be found in $[10,14,28]$. Most of the resolution techniques are fundamentally based on two principles of using the ADM. The first approach is the method of undetermined coefficients (see, e.g., $[10,14,29,30]$ ) in the ADM which approximates the constants of integration embedded in the recursion scheme of the ADM by solving numerically a sequence of nonlinear algebraic equations obtained by employing boundary conditions. The Duan-Rach modified ADM (see, e.g., [26, $27,30,31]$ ), which is the second approach, determines the remaining unknown constants of integration of the solution by using the remaining boundary conditions before designing a suitable modified recursion scheme. In the Duan-Rach modified ADM, the constants of integration are calculated simultaneously along with the solution components. Details of the Duan-Rach modified ADM will be discussed in Section 2.3.

In our work, we study the use of the Duan-Rach modified ADM to solve nonlinear high fractional order boundary value problems with a variety of boundary conditions such as Robin and separated boundary conditions. We will study these fractional BVPs for the Caputo fractional derivative which allows some boundary conditions to be included into the formulas of the solutions. To the best of the authors' knowledge, our paper will be the first to develop formulas for the recursion schemes obtained by using the Duan-Rach modified ADM for the above types of problems.
This paper is organized as follows. In Section 2, we review necessary definitions and important properties of the fractional order integrals and derivatives that are needed in our work. The principal reviews of the ADM and the DuanRach modified ADM are briefly given in this section as well. In Section 3, we give the formulas for the recursion schemes obtained by using the Duan-Rach modified ADM for selected types of nonlinear fractional BVPs. In Section 4, we give numerical examples of solutions obtained using the proposed recursion schemes for some scientific fractional BVPs with mixed sets of Dirichlet, Neumann, Robin, and separated boundary conditions. These examples include a Bratu-type fractional BVP, an oscillating base temperature equation, and an elastic beam problem.

\section{Mathematical Preliminaries}

2.1. Review of Fractional Order Integrals and Derivatives. In this section, we present basic definitions and important theorems of the fractional calculus (see [5, 32-35]) required in this paper.

Definition 1 (see [5]). A function $f(x)(x>0)$ is said to be in the space $C_{\alpha}(\alpha \in \mathbb{R})$ if it can be expressed as $f(x)=x^{p} f_{1}(x)$ for some $p>\alpha$, where $f_{1}(x)$ is continuous in $[0, \infty)$, and it is said to be in the space $C_{\alpha}^{m}$ if $f^{(m)} \in C_{\alpha}, m \in \mathbb{N}$. Clearly, $C_{\alpha} \subseteq C_{\beta}$ if $\beta, \alpha \in \mathbb{R}$ and $\beta \leq \alpha$.

Definition 2 (see [5]). The Riemann-Liouville fractional integral operator of order $\alpha>0$ of a function $f \in C_{\alpha}$ with $a \geq 0$ is defined as

$$
\begin{aligned}
& \mathrm{RL}_{a}^{\alpha} f(x)=\frac{1}{\Gamma(\alpha)} \int_{a}^{x}(x-\tau)^{\alpha-1} f(\tau) d \tau, \quad x>a, \\
& \mathrm{RL} J_{a}^{0} f(x)=f(x),
\end{aligned}
$$

where $\Gamma(\cdot)$ is the gamma function.

Definition 3 (see [5]). The Caputo fractional derivative of $f(x)$ of order $\alpha>0$ with $a \geq 0$ is defined as

$$
{ }_{C} D_{a}^{\alpha} f(x)= \begin{cases}\mathrm{RL}_{a} J_{a}^{m-\alpha} f^{(m)}(x)=\frac{1}{\Gamma(m-\alpha)} \int_{a}^{x} \frac{f^{(m)}(\tau)}{(x-\tau)^{\alpha-m+1}} d \tau, & m-1<\alpha<m, m \in \mathbb{N} \\ f^{(m)}(x), & \alpha=m,\end{cases}
$$

for $x \geq a$ and $f \in C_{-1}^{m}$.

For $0<\beta \leq \alpha, a \geq 0$, and $m \in \mathbb{N}$, we have the following important properties [5]:

$$
\begin{array}{r}
\mathrm{RL}_{a \mathrm{RL}} J_{a}^{\alpha} f(x)={ }_{\mathrm{RL}} J_{a \mathrm{RL}}^{\beta} J_{a}^{\alpha} f(x)={ }_{\mathrm{RL}} J_{a}^{\alpha+\beta} f(x), \\
\text { where } f \in C_{\alpha},
\end{array}
$$

$$
{ }_{\mathrm{RL}} J_{a}^{\alpha}(x-a)^{\mu}=\frac{\Gamma(\mu+1)}{\Gamma(\mu+\alpha+1)}(x-a)^{\mu+\alpha},
$$

where $\mu>-1, x>a$,

$$
{ }_{C} D_{a}^{\alpha}(x-a)^{\mu}=\frac{\Gamma(\mu+1)}{\Gamma(\mu-\alpha+1)}(x-a)^{\mu-\alpha},
$$

where $0 \leq m-1<\alpha \leq m<\mu+1, \mu>0, x>a$, 


$$
\mathrm{RL}_{a C} J_{a}^{\gamma} D_{a}^{\gamma} f(x)=f(x)-\sum_{k=0}^{m-1} f^{(k)}(a) \frac{(x-a)^{k}}{k !}
$$

$$
\text { where } m-1<\gamma \leq m, f \in C_{\gamma}^{m}, \gamma \geq-1 \text {. }
$$

2.2. Review of the Decomposition Methods. We first review the important concepts of the Adomian decomposition method (ADM) [9] introduced by George Adomian who is an American physicist. The ADM combined with the use of symbolic algebra packages such as MATHEMATICA or MAPLE is a powerful method for solving nonlinear operator equations including ordinary or partial differential equations [14, 36]. Here we describe the ADM to solve integer order IVPs and BVPs as follows.

Consider the ordinary differential equation in the following operator form:

$$
L u+R u+N u=g
$$

where $L$ is the highest order derivative; that is, $L=$ $\left(d^{p} / d x^{p}\right)(\cdot)$, where $p$ is the integer order of the derivative, which is assumed to be easily invertible, $R$ is a linear differential operator of order less than $L, N$ denotes a nonlinear operator assumed to be analytic, $g$ is a source term, and $u$ is the solution of the equation. The ADM decomposes the solution $u(x)$ and the analytic nonlinear term $N u$ of the nonlinear operator equation (7) into a rapidly convergent series of solution components and a series of the Adomian polynomials.

Applying the inverse linear operator $L^{-1}$, which is a $p$ fold definite integration, to both sides of (7) and using the given conditions, that is, the initial conditions or boundary conditions, and the fact that $L^{-1} L u=u-\Phi$, we obtain

$$
u=\Phi+L^{-1} g-L^{-1}(R u)-L^{-1}(N u)
$$

where $\Phi$ denotes the terms arising from using the given conditions. The ADM decomposes the solution $u(x)$ into an infinite series

$$
u=\sum_{n=0}^{\infty} u_{n}
$$

and then it decomposes the nonlinear term $N u(x)$ into a series

$$
N u=\sum_{n=0}^{\infty} A_{n}
$$

where $A_{n}=A_{n}\left(u_{0}(x), u_{1}(x), \ldots, u_{n}(x)\right)$ are the Adomian polynomials that are obtained by the following formula (see the derivation of the formula in $[27,37]$ and the references therein):

$$
A_{n}=\left.\frac{1}{n !} \frac{d^{n}}{d \lambda^{n}} N\left(\sum_{k=0}^{\infty} u_{k} \lambda^{k}\right)\right|_{\lambda=0}, \quad n \geq 0,
$$

where $\lambda$ is a grouping parameter. The first six Adomian polynomials obtained by using (11) for the general analytic nonlinear term $N u(x)=f(u(x))$ are as follows:

$$
\begin{aligned}
A_{0}= & f\left(u_{0}\right), \\
A_{1}= & f^{\prime}\left(u_{0}\right) u_{1}, \\
A_{2}= & f^{\prime}\left(u_{0}\right) u_{2}+f^{\prime \prime}\left(u_{0}\right) \frac{u_{1}^{2}}{2 !}, \\
A_{3}= & f^{\prime}\left(u_{0}\right) u_{3}+f^{\prime \prime}\left(u_{0}\right) u_{1} u_{2}+f^{\prime \prime \prime}\left(u_{0}\right) \frac{u_{1}^{3}}{3 !}, \\
A_{4}= & f^{\prime}\left(u_{0}\right) u_{4}+f^{\prime \prime}\left(u_{0}\right)\left(\frac{u_{2}^{2}}{2 !}+u_{1} u_{3}\right) \\
& +f^{\prime \prime \prime}\left(u_{0}\right) \frac{u_{1}^{2} u_{2}}{2 !}+f^{(4)}\left(u_{0}\right) \frac{u_{1}^{4}}{4 !}, \\
A_{5}= & f^{\prime}\left(u_{0}\right) u_{5}+f^{\prime \prime}\left(u_{0}\right)\left(u_{2} u_{3}+u_{1} u_{4}\right) \\
& +f^{(3)}\left(u_{0}\right)\left(\frac{u_{3} u_{1}^{2}}{2 !}+\frac{u_{2}^{2} u_{1}}{2 !}\right) \\
& +f^{(4)}\left(u_{0}\right) \frac{u_{2} u_{1}^{3}}{3 !}+f^{(5)}\left(u_{0}\right) \frac{u_{1}^{5}}{5 !} .
\end{aligned}
$$

We observe that the Adomian polynomials are of the following forms:

$$
\begin{aligned}
& A_{0}=f\left(u_{0}\right), \\
& A_{n}=\sum_{k=1}^{n} f^{(k)}\left(u_{0}\right) C_{n}^{k}, \quad n \geq 1,
\end{aligned}
$$

where $C_{n}^{k}$ are the sums of all possible products of $k$ components from $u_{1}, u_{2}, \ldots, u_{n-k+1}$, whose subscripts sum to $n$, divided by the factorial of the number of repeated subscripts.

From (8), (9), and (10), we have

$$
\sum_{n=0}^{\infty} u_{n}=\Phi+L^{-1} g-L^{-1}(R u)-L^{-1}(N u)
$$

and the classic (or standard) Adomian recursion scheme [36, 38 ] is as follows:

$$
\begin{aligned}
u_{0}(x) & =\Phi+L^{-1} g, \\
u_{n+1}(x) & =-L^{-1}\left(R u_{n}\right)-L^{-1}\left(A_{n}\right), \quad n \geq 0 .
\end{aligned}
$$

Then the $n$-term approximation of the solution is

$$
\varphi_{n}(x)=\sum_{k=0}^{n-1} u_{k}(x)
$$

which in the $\lim _{n \rightarrow \infty}$ yields the exact solution to (7) as

$$
u(x)=\lim _{n \rightarrow \infty} \varphi_{n}(x) .
$$


The choice of different initial solutions $u_{0}(x)$ can generate different recursion schemes which can remedy problems in the classic scheme caused by the difficulty of integration for $u_{0}(x)$ or the slowness of convergence to the series solution (see [39] for details). One criterion that can be used for choosing the initial solution is to achieve a simple integration for the initial solution component of the series solution. Then, to obtain a fast rate of convergence and an extended region of convergence, we can apply Duan's convergence parameter technique [40-42] to the recursion scheme for that initial solution component. Duan and coresearchers [26, 27] have shown that the parametrized recursion scheme usually gives a sequence of decreasing maximal errors which approach zero when the value of $n$ in the approximation $\varphi_{n}(x)$ increases.

2.3. The Duan-Rach Modified Decomposition Method. In this section, we will provide the idea of the modified ADM called the Duan-Rach modified decomposition method to solve integer order BVPs. The method generates a recursion scheme for computing successive solution components without any undetermined coefficients. Unlike the method of undetermined coefficients, this new Duan-Rach modified decomposition method $[26,27,30]$ does not require the solution of a sequence of the nonlinear algebraic equations obtained from the approximation $\varphi_{n}(x)$ for the constants of integration. In the Duan-Rach modified decomposition method, we first incorporate as many of the given boundary conditions as possible into the solution $u(x)$ in (8) of the BVP and then we determine the remaining unknown constants of integration before constructing the modified recursion scheme.

We will now give an example of the use of the Duan-Rach modified decomposition method to solve the following twopoint BVP:

$$
\begin{aligned}
L u+R u+N u & =g, \\
u(a) & =\beta, \\
u(b) & =\gamma,
\end{aligned}
$$

where the operator $L$ in $(18)$ is $L=\left(d^{2} / d x^{2}\right)(\cdot)$. Using the first condition of the boundary conditions in (18), we obtain the solution form as follows:

$$
\begin{aligned}
u(x)= & \beta+u^{\prime}(a)(x-a)+L^{-1} g-L^{-1}(R u) \\
& -L^{-1}(N u)
\end{aligned}
$$

where $L^{-1}=\int_{a}^{x} \int_{a}^{\tau}(\cdot) d s d \tau$. We then apply the second condition of the boundary conditions in (18) to solve for $u^{\prime}(a)$ which can be expressed as follows:

$$
\begin{aligned}
& u^{\prime}(a)=\frac{1}{b-a}\left[\gamma-\beta-\left[L^{-1} g\right]_{x=b}+\left[L^{-1}(R u)\right]_{x=b}\right. \\
& \left.+\left[L^{-1}(N u)\right]_{x=b}\right] .
\end{aligned}
$$

Substituting (20) into (19) yields

$$
\begin{aligned}
u(x)= & \beta+\frac{\gamma-\beta}{b-a}(x-a)-\frac{x-a}{b-a}\left[L^{-1} g\right]_{x=b} \\
& +\frac{x-a}{b-a}\left[L^{-1}(R u)\right]_{x=b} \\
& +\frac{x-a}{b-a}\left[L^{-1}(N u)\right]_{x=b}+L^{-1} g-L^{-1}(R u) \\
& -L^{-1}(N u) .
\end{aligned}
$$

Applying the Adomian polynomials for the nonlinear terms in (21), we then obtain the following modified recursion scheme:

$$
\begin{aligned}
u_{0}(x)= & \beta+\frac{\gamma-\beta}{b-a}(x-a)-\frac{x-a}{b-a}\left[L^{-1} g\right]_{x=b} \\
& +L^{-1} g \\
u_{n+1}(x)= & \frac{x-a}{b-a}\left[L^{-1}(R u)\right]_{x=b} \\
& +\frac{x-a}{b-a}\left[L^{-1}\left(A_{n}\right)\right]_{x=b}-L^{-1}(R u) \\
& -L^{-1}\left(A_{n}\right), \quad n \geq 0 .
\end{aligned}
$$

\section{Description of the Proposed Recursion Schemes for Solving Certain Types of Fractional Order BVPs}

The main advantage of using the Duan-Rach modified ADM for solving nonlinear integer order BVPs which we can see from Section 2.3 is that evaluating the inverse operator directly at the boundary points allows us to obtain the solution components without using numerical methods to calculate the values of unknown constants of integration as in the method of undetermined coefficients. In this section, we construct the recursion schemes developed by using the Duan-Rach modified ADM for solving fractional higher order two-point BVPs with their boundary conditions. We give examples for a set of Robin conditions and separated boundary conditions. We consider the following nonlinear fractional order differential equation:

$$
{ }_{C} D_{a}^{\alpha} u(x)+N u=g(x), \quad a \leq x \leq b,
$$

where $\alpha$ is a fractional order of the differential equation with $m-1 \leq \alpha \leq m, m=2,4, N$ is a nonlinear operator, $g(x)$ is a source term, and $u(x)$ is the solution of the equation. Comparing with (7), we see that the operator $L={ }_{C} D_{a}^{\alpha}$ is the Caputo fractional derivative operator of order $\alpha$. Applying $L^{-1}={ }_{\mathrm{RL}} J_{a}^{\alpha}$, which is the Riemann-Liouville fractional 
integral operator of order $\alpha$, to both sides of (23) and using the property in (6) yield

$$
\begin{aligned}
& \mathrm{RL}_{a C} J_{a}^{\alpha} D_{a}^{\alpha} u(x)={ }_{\mathrm{RL}} J_{a}^{\alpha}(g(x)-N u), \\
& u(x)-\sum_{k=0}^{m-1} u^{(k)}(a) \frac{(x-a)^{k}}{k !} \\
& ={ }_{\mathrm{RL}} J_{a}^{\alpha}(g(x))-{ }_{\mathrm{RL}} J_{a}^{\alpha}(N u), \\
& u(x) \\
& =\sum_{k=0}^{m-1} u^{(k)}(a) \frac{(x-a)^{k}}{k !}+{ }_{\mathrm{RL}} J_{a}^{\alpha}(g(x)) \\
& -{ }_{\mathrm{RL}} J_{a}^{\alpha}(N u) .
\end{aligned}
$$

The specific types of boundary conditions imposed on the fractional differential equation in (23) or the fractional integral equation in (24) depend upon the value of $\alpha$.

3.1. The Fractional Order Differential Equation (23) with a Set of Robin Boundary Conditions. We consider a nonlinear fractional order differential equation of the form

$$
{ }_{C} D_{a}^{\alpha} u+N u=g(x), \quad a \leq x \leq b, 1<\alpha \leq 2,
$$

subject to a set of Robin boundary conditions

$$
\begin{gathered}
p u(a)+q u^{\prime}(a)=\beta, \\
r u(b)+s u^{\prime}(b)=\gamma,
\end{gathered}
$$

where $N(u(x))$ is an analytic nonlinear term and $p, q, r$, and $s$ satisfy the following condition:

$$
p s-q r+p r(b-a) \neq 0 .
$$

In order to have the two boundary conditions required for the problem and to make the condition (28) hold, it is necessary to have $p, q$ not both zero, $r, s$ not both zero, and $p, r$ not both zero.

From (24), we have that the solution of this BVP can be written in the form

$$
\begin{aligned}
u(x)= & u(a)+u^{\prime}(a)(x-a)+{ }_{\mathrm{RL}} J_{a}^{\alpha}(g(x)) \\
& -{ }_{\mathrm{RL}} J_{a}^{\alpha}(N u) .
\end{aligned}
$$

We now apply the Duan-Rach modified ADM to the problem in (25). Using (29), we evaluate $u(x)$ at $x=b$ to obtain

$$
\begin{aligned}
u(b)= & u(a)+u^{\prime}(a)(b-a)+\left[{ }_{\mathrm{RL}} J_{a}^{\alpha}(g(x))\right]_{x=b} \\
& -\left[{ }_{\mathrm{RL}} J_{a}^{\alpha}(N u)\right]_{x=b},
\end{aligned}
$$

where $\left.{ }_{\mathrm{RL}} J_{a}^{\alpha}(\cdot)\right]_{x=b}$ is the Riemann-Liouville fractional integral operator of order $\alpha$ evaluated at $x=b$.
Differentiating (29) and then using the property that $(d / d x)_{\mathrm{RL}} J_{a}^{\alpha}(\cdot)={ }_{\mathrm{RL}} J_{a}^{\alpha-1}(\cdot)$ and evaluating $u^{\prime}(x)$ at $x=b$, we obtain

$$
\begin{aligned}
u^{\prime}(b)= & u^{\prime}(a)+\left[{ }_{\mathrm{RL}} J_{a}^{\alpha-1}(g(x))\right]_{x=b} \\
& -\left[\mathrm{RL}_{a}^{\alpha-1}(N u)\right]_{x=b},
\end{aligned}
$$

where $\left[{ }_{\mathrm{RL}} J_{a}^{\alpha-1}(\cdot)\right]_{x=b}$ is the Riemann-Liouville fractional integral operator of order $\alpha-1$ evaluated at $x=b$.

Substituting (30) and (31) into (27), we get

$$
\begin{aligned}
r & {\left[u(a)+u^{\prime}(a)(b-a)+\left[{ }_{\mathrm{RL}} J_{a}^{\alpha}(g(x))\right]_{x=b}\right.} \\
& \left.-\left[{ }_{\mathrm{RL}} J_{a}^{\alpha}(N u)\right]_{x=b}\right]+s\left[u^{\prime}(a)\right. \\
& \left.+\left[\mathrm{RL}_{a}^{\alpha-1}(g(x))\right]_{x=b}-\left[\mathrm{RL}_{a} J_{a}^{\alpha-1}(N u)\right]_{x=b}\right]=\gamma .
\end{aligned}
$$

After manipulating the terms in the above equation, we obtain

$$
\begin{aligned}
r u(a) & +[r(b-a)+s] u^{\prime}(a) \\
= & \gamma+r\left(\left[\mathrm{RL}_{a}^{\alpha}(N u)\right]_{x=b}-\left[\mathrm{RL}_{a}^{\alpha}(g(x))\right]_{x=b}\right) \\
& +s\left(\left[\mathrm{RL}_{a}^{\alpha-1}(N u)\right]_{x=b}-\left[{ }_{\mathrm{RL}} J_{a}^{\alpha-1}(g(x))\right]_{x=b}\right) .
\end{aligned}
$$

It is possible to solve the system of two linearly independent equations (26) and (33) for the two remaining undetermined coefficients $u(a)$ and $u^{\prime}(a)$ if the determinant of the coefficient matrix denoted by $\Delta$ is not zero, that is, if

$$
\Delta=\left|\begin{array}{cc}
p & q \\
r & s+r(b-a)
\end{array}\right|=p s-q r+p r(b-a) \neq 0 .
$$

For $\Delta \neq 0$, the values of $u(a)$ and $u^{\prime}(a)$ can be expressed in terms of the specified values of the system parameters $a, b, \beta$, $\gamma, p, q, r$, and $s$ as follows:

$$
\begin{aligned}
& u(a)=\frac{1}{\Delta}[r \beta(b-a)+s \beta-q r \\
& +q s\left[{ }_{\mathrm{RL}} J_{a}^{\alpha-1}(g(x))\right]_{x=b}+q r\left[{ }_{\mathrm{RL}} J_{a}^{\alpha}(g(x))\right]_{x=b} \\
& \left.\quad-q s\left[{ }_{\mathrm{RL}} J_{a}^{\alpha-1}(N u)\right]_{x=b}-q r\left[{ }_{\mathrm{RL}} J_{a}^{\alpha}(N u)\right]_{x=b}\right], \\
& u^{\prime}(a)=\frac{1}{\Delta}\left[p \gamma-r \beta-p s\left[{ }_{\mathrm{RL}} J_{a}^{\alpha-1}(g(x))\right]_{x=b}\right. \\
& \quad-\operatorname{pr}\left[{ }_{\mathrm{RL}} J_{a}^{\alpha}(g(x))\right]_{x=b}+p s\left[{ }_{\mathrm{RL}} J_{a}^{\alpha-1}(N u)\right]_{x=b} \\
& \left.+\operatorname{pr}\left[{ }_{\mathrm{RL}} J_{a}^{\alpha}(N u)\right]_{x=b}\right] .
\end{aligned}
$$


Substituting (35) into (29), we obtain the following equivalent nonlinear Fredholm-Volterra integral equation:

$$
\begin{aligned}
u(x) & =\frac{1}{\Delta}[s \beta-q \gamma+r \beta(b-a)+(p \gamma-r \beta)(x-a) \\
+ & (q s-p s(x-a))\left[{ }_{\mathrm{RL}} J_{a}^{\alpha-1}(g(x))\right]_{x=b} \\
+ & (q r-p r(x-a))\left[\mathrm{RL}_{a} J_{a}^{\alpha}(g(x))\right]_{x=b} \\
& -(q s-p s(x-a))\left[\mathrm{RL}_{a}^{\alpha-1}(N u)\right]_{x=b} \\
& \left.-(q r-p r(x-a))\left[\mathrm{RL}_{a}^{\alpha} J_{a}^{\alpha}(N u)\right]_{x=b}\right] \\
& +{ }_{\mathrm{RL}} J_{a}^{\alpha}(g(x))-{ }_{\mathrm{RL}} J_{a}^{\alpha}(N u),
\end{aligned}
$$

which is free of any undetermined coefficients. Therefore (36) represents the solution of the fractional order nonlinear differential equation (25) subject to the Robin boundary conditions (26) and (27). Next we apply the decomposition to the solution $u(x)$ and the nonlinear term $N u(x)$; that is,

$$
\begin{gathered}
u(x)=\sum_{n=0}^{\infty} u_{n}(x), \\
N u(x)=\sum_{n=0}^{\infty} A_{n}(x),
\end{gathered}
$$

respectively, where $A_{n}(x)$ are the Adomian polynomials defined in (11).

Inserting the equations in (37) into (36), the solution components are determined by the following modified recursion scheme:

$$
\begin{aligned}
& u_{0}(x)=\frac{1}{\Delta}[s \beta-q \gamma+r \beta(b-a)+(p \gamma-r \beta)(x-a) \\
& +(q s-p s(x-a))\left[{ }_{\mathrm{RL}} J_{a}^{\alpha-1}(g(x))\right]_{x=b} \\
& \left.+(q r-p r(x-a))\left[{ }_{\mathrm{RL}} J_{a}^{\alpha}(g(x))\right]_{x=b}\right] \\
& \quad+{ }_{\mathrm{RL}} J_{a}^{\alpha}(g(x)), \\
& u_{n+1}(x)=\frac{1}{\Delta}\left[(-q s+p s(x-a))\left[{ }_{\mathrm{RL}} J_{a}^{\alpha-1}\left(A_{n}\right)\right]_{x=b}\right. \\
& \left.\quad+(-q r+p r(x-a))\left[{ }_{\mathrm{RL}} J_{a}^{\alpha}\left(A_{n}\right)\right]_{x=b}\right] \\
& \quad-{ }_{\mathrm{RL}} J_{a}^{\alpha}\left(A_{n}\right), \quad n \geq 0,
\end{aligned}
$$

where the resulting integrals are assumed to exist.

The $n$-term approximation of the solution to the BVP obtained by the ADM is the following truncated decomposition series:

$$
\varphi_{n}(x)=\sum_{k=0}^{n-1} u_{k}(x)
$$

With the above decomposition obtained by the Duan-Rach modified ADM, each approximation $\varphi_{n}(x), n \geq 1$, must exactly satisfy the boundary conditions (26) and (27). In addition, other techniques such as partitioning initial terms into two appropriate terms $[39,43,44]$ or using the Duan's convergence parameter [40-42] can be incorporated, if necessary, into the recursion scheme (38) for solving the BVP described in (25)-(27).

Using the general formulas given above, we can derive the equivalent nonlinear Fredholm-Volterra integral equations and their associated recursion schemes for (25) for the special cases of the boundary conditions in (26) and (27). The results are as follows.

Case 1. The nonlinear fractional BVP consists of (25) and the following Dirichlet boundary conditions:

$$
\begin{aligned}
& u(a)=\beta, \\
& u(b)=\gamma .
\end{aligned}
$$

The boundary conditions (40) correspond to the case of $p=$ $r=1$ and $q=s=0$ in (26) and (27). Thus we have $\Delta=b-a \neq$ 0 and then (36) is reduced to

$$
\begin{aligned}
& u(x)=\frac{1}{b-a}[\beta(b-a)+(\gamma-\beta)(x-a) \\
& \quad-(x-a)\left[{ }_{\mathrm{RL}} J_{a}^{\alpha}(g(x))\right]_{x=b} \\
& \left.\quad+(x-a)\left[\mathrm{RL}_{a}^{\alpha}(N u)\right]_{x=b}\right]+{ }_{\mathrm{RL}} J_{a}^{\alpha}(g(x)) \\
& \quad-\mathrm{RL}_{a} J_{a}^{\alpha}(N u),
\end{aligned}
$$

where $\mathrm{RL}_{a} J_{a}^{\alpha}(\cdot)$ and $\left[{ }_{\mathrm{RL}} J_{a}^{\alpha}(\cdot)\right]_{x=b}$ are the Riemann-Liouville fractional integral operator of order $\alpha$ and the RiemannLiouville fractional integral operator of order $\alpha$ evaluated at $x=b$, respectively.

Substituting equations in (37) into (41), we can determine the solution components from the following modified recursion scheme:

$$
\begin{aligned}
& u_{0}(x)=\frac{1}{b-a}[\beta(b-a)+(\gamma-\beta)(x-a) \\
& \left.-(x-a)\left[{ }_{\mathrm{RL}} J_{a}^{\alpha}(g(x))\right]_{x=b}\right]+{ }_{\mathrm{RL}} J_{a}^{\alpha}(g(x)), \\
& u_{n+1}(x)=\frac{x-a}{b-a}\left[{ }_{\mathrm{RL}} J_{a}^{\alpha}\left(A_{n}\right)\right]_{x=b}-\mathrm{RL}_{a} J_{a}^{\alpha}\left(A_{n}\right),
\end{aligned}
$$

$n \geq 0$,

provided that the resulting integrals exist.

Case 2. The nonlinear fractional BVP consists of (25) and the following mixed set of Neumann and Dirichlet boundary conditions:

$$
\begin{gathered}
u^{\prime}(a)=\beta, \\
u(b)=\gamma .
\end{gathered}
$$


The boundary conditions (43) correspond to the case of $p=$ $s=0$ and $q=r=1$ in (26) and (27). Thus we have $\Delta=-1 \neq 0$ and then (36) becomes

$$
\begin{aligned}
u(x)= & \gamma-\beta(b-a)+\beta(x-a)-\left[{ }_{\mathrm{RL}} J_{a}^{\alpha}(g(x))\right]_{x=b} \\
& +\left[{ }_{\mathrm{RL}} J_{a}^{\alpha}(N u)\right]_{x=b}+{ }_{\mathrm{RL}} J_{a}^{\alpha}(g(x)) \\
& -{ }_{\mathrm{RL}} J_{a}^{\alpha}(N u),
\end{aligned}
$$

where $\mathrm{RL}_{a} J_{a}^{\alpha}(\cdot)$ and $\left[{ }_{\mathrm{RL}} J_{a}^{\alpha}(\cdot)\right]_{x=b}$ are the Riemann-Liouville fractional integral operator of order $\alpha$ and the RiemannLiouville fractional integral operator of order $\alpha$ evaluated at $x=b$, respectively.

Substituting the equations in (37) into (44), we obtain the following modified recursion scheme:

$$
\begin{aligned}
u_{0}(x)= & \gamma-\beta(b-a)+\beta(x-a) \\
& -\left[{ }_{\mathrm{RL}} J_{a}^{\alpha}(g(x))\right]_{x=b}+{ }_{\mathrm{RL}} J_{a}^{\alpha}(g(x)), \\
u_{n+1}(x)= & {\left[{ }_{\mathrm{RL}} J_{a}^{\alpha}\left(A_{n}\right)\right]_{x=b}-{ }_{\mathrm{RL}} J_{a}^{\alpha}\left(A_{n}\right), \quad n \geq 0, }
\end{aligned}
$$

where we assume the resulting integrals exist.

Case 3. The nonlinear fractional BVP consists of (25) and the following mixed set of Robin and Neumann boundary conditions:

$$
\begin{aligned}
p u(a)+q u^{\prime}(a) & =\beta, \\
s u^{\prime}(b) & =\gamma .
\end{aligned}
$$

The boundary conditions (46) correspond to the case of $r=0$ and $s=1$ in (27). Thus we have $\Delta=p \neq 0$ and then $\mathrm{E}$ (36) is reduced to

$$
\begin{aligned}
& u(x)=\frac{1}{p}[\beta-q \gamma+p \gamma(x-a) \\
& +(q-p(x-a))\left[\mathrm{RL}_{a}^{\alpha-1}(g(x))\right]_{x=b} \\
& \left.+(-q+p(x-a))\left[\mathrm{RL}_{a}^{\alpha-1}(N u)\right]_{x=b}\right] \\
& +\mathrm{RL}_{a}^{\alpha} J_{a}^{\alpha}(g(x))-\mathrm{RL}_{a}^{\alpha}(N u),
\end{aligned}
$$

where ${ }_{\mathrm{RL}} J_{a}^{\alpha}(\cdot)$ and $\left[{ }_{\mathrm{RL}} J_{a}^{\alpha-1}(\cdot)\right]_{x=b}$ are the Riemann-Liouville fractional integral operator of order $\alpha$ and the RiemannLiouville fractional integral operator of order $\alpha-1$ evaluated at $x=b$, respectively.

Insertion of the equations in (37) into (47) gives the following modified recursion scheme:

$$
\begin{aligned}
& u_{0}(x)=\frac{1}{p}[\beta-q \gamma+p \gamma(x-a) \\
& \left.+(q-p(x-a))\left[\mathrm{RL}_{a}^{\alpha-1}(g(x))\right]_{x=b}\right] \\
& +{ }_{\mathrm{RL}} J_{a}^{\alpha}(g(x)),
\end{aligned}
$$

$$
\begin{aligned}
& u_{n+1}(x)=\frac{1}{p}\left[(-q+p(x-a))\left[{ }_{\mathrm{RL}} J_{a}^{\alpha-1}\left(A_{n}\right)\right]_{x=b}\right] \\
& -{ }_{\mathrm{RL}} J_{a}^{\alpha}\left(A_{n}\right), \quad n \geq 0,
\end{aligned}
$$

where we assume the resulting integrals exist.

3.2. The Fractional Order Differential Equation (23) with Separated Boundary Conditions. We consider a nonlinear fractional order two-point BVP consisting of the fractional order differential equation

$$
{ }_{C} D_{a}^{\alpha} u+N u=g(x), \quad a \leq x \leq b, 3<\alpha \leq 4,
$$

and the following separated boundary conditions:

$$
\begin{aligned}
u(a) & =\rho, \\
p u^{\prime \prime}(a)-q u^{\prime \prime \prime}(a) & =\beta, \\
u(b) & =\sigma, \\
r u^{\prime \prime}(b)+s u^{\prime \prime \prime}(b) & =\gamma,
\end{aligned}
$$

where $N(u(x))$ is an analytic nonlinear term, $\rho, \sigma, \beta, \gamma \in \mathbb{R}$, and $p, q, r, s \geq 0$ satisfy the following condition

$$
p s+q r+\operatorname{pr}(b-a) \neq 0 \text {. }
$$

In order to satisfy four necessary boundary conditions required for the problem and to have condition (52), it is necessary to have $p, q$ not both zero, $r, s$ not both zero, and $p, r$ not both zero.

From (24) and the first condition in (50), we have that the solution of this BVP can be written in the form

$$
\begin{aligned}
u(x)= & u(a)+u^{\prime}(a)(x-a)+\frac{u^{\prime \prime}(a)}{2}(x-a)^{2} \\
& +\frac{u^{\prime \prime \prime}(a)}{6}(x-a)^{3}+{ }_{\mathrm{RL}} J_{a}^{\alpha}(g(x)) \\
& -\mathrm{RL}_{a}^{\alpha}(N u), \\
u(x)= & \rho+u^{\prime}(a)(x-a)+\frac{u^{\prime \prime}(a)}{2}(x-a)^{2} \\
& +\frac{u^{\prime \prime \prime}(a)}{6}(x-a)^{3}+{ }_{\mathrm{RL}} J_{a}^{\alpha}(g(x)) \\
& -\mathrm{RL}_{a}^{\alpha}(N u) .
\end{aligned}
$$

We now apply the Duan-Rach modified ADM to the problem in (49). Using (53) and the first condition in (51), we evaluate $u(x)$ at $x=b$ to obtain

$$
\begin{aligned}
\sigma= & u(b) \\
= & \rho+u^{\prime}(a)(b-a)+\frac{u^{\prime \prime}(a)}{2}(b-a)^{2} \\
& +\frac{u^{\prime \prime \prime}(a)}{6}(b-a)^{3}+\left[{ }_{\mathrm{RL}} J_{a}^{\alpha}(g(x))\right]_{x=b} \\
& -\left[{ }_{\mathrm{RL}} J_{a}^{\alpha}(N u)\right]_{x=b} .
\end{aligned}
$$


Differentiating (53) three times and then using the properties that $\left(d^{n} / d x^{n}\right){ }_{\mathrm{RL}} J_{a}^{\alpha}(\cdot)={ }_{\mathrm{RL}} J_{a}^{\alpha-n}(\cdot), n=1,2,3$, to the resulting equations, we obtain

$$
\begin{aligned}
u^{\prime}(x)= & u^{\prime}(a)+u^{\prime \prime}(a)(x-a)+\frac{u^{\prime \prime \prime}(a)}{2}(x-a)^{2} \\
& +{ }_{\mathrm{RL}} J_{a}^{\alpha-1}(g(x))-\mathrm{RL}_{a} J_{a}^{\alpha-1}(N u), \\
u^{\prime \prime}(x)= & u^{\prime \prime}(a)+u^{\prime \prime \prime}(a)(x-a)+\mathrm{RL}_{a}^{\alpha-2}(g(x)) \\
& -\mathrm{RL}_{a}^{\alpha-2}(N u), \\
u^{\prime \prime \prime}(x)= & u^{\prime \prime \prime}(a)+{ }_{\mathrm{RL}} J_{a}^{\alpha-3}(g(x))-{ }_{\mathrm{RL}} J_{a}^{\alpha-3}(N u) .
\end{aligned}
$$

$$
\begin{aligned}
u^{\prime \prime \prime}(b)= & u^{\prime \prime \prime}(a)+\left[{ }_{\mathrm{RL}} J_{a}^{\alpha-3}(g(x))\right]_{x=b} \\
& -\left[{ }_{\mathrm{RL}} J_{a}^{\alpha-3}(\mathrm{Nu})\right]_{x=b} .
\end{aligned}
$$

Insertion of (56) into (51) gives the following relation:

$$
\begin{aligned}
& r u^{\prime \prime}(a)+(r(b-a)+s) u^{\prime \prime \prime}(a) \\
& =\gamma+r\left(\left[\mathrm{RL}_{a}^{\alpha-2}(N u)\right]_{x=b}-\left[{ }_{\mathrm{RL}} J_{a}^{\alpha-2}(g(x))\right]_{x=b}\right) \\
& \quad+s\left(\left[\mathrm{RL}_{a}^{\alpha-3}(N u)\right]_{x=b}-\left[\mathrm{RL}_{a}^{\alpha-3}(g(x))\right]_{x=b}\right) .
\end{aligned}
$$

It is possible to solve the system consisting of the second condition of (50) and (57) for the two remaining undetermined coefficients $u^{\prime \prime}(a)$ and $u^{\prime \prime \prime}(a)$ if the determinant of the coefficient matrix denoted by $\Delta$ is not zero, that is, if

$$
\Delta=\left|\begin{array}{cc}
p & -q \\
r & r(b-a)+s
\end{array}\right|=p s+q r+p r(b-a) \neq 0 .
$$

For $\Delta \neq 0$, the values of $u^{\prime \prime}(a)$ and $u^{\prime \prime \prime}(a)$ can be expressed in terms of the specified values of the system parameters $a, b, \beta$, $\gamma, p, q, r$, and $s$ as follows:

$$
\begin{aligned}
& u^{\prime \prime}(a)=\frac{1}{\Delta}[r \beta(b-a)+s \beta+q \gamma \\
& +q r\left(\left[{ }_{\mathrm{RL}} J_{a}^{\alpha-2}(N u)\right]_{x=b}-\left[\mathrm{RL}_{a}^{\alpha-2}(g(x))\right]_{x=b}\right) \\
& \left.+q s\left(\left[{ }_{\mathrm{RL}} J_{a}^{\alpha-3}(N u)\right]_{x=b}-\left[{ }_{\mathrm{RL}} J_{a}^{\alpha-3}(g(x))\right]_{x=b}\right)\right], \\
& u^{\prime \prime \prime}(a)=\frac{1}{\Delta}[p \gamma-r \beta \\
& +\operatorname{pr}\left(\left[\mathrm{RL}_{a} J_{a}^{\alpha-2}(N u)\right]_{x=b}-\left[\mathrm{RL}_{a}^{\alpha-2}(g(x))\right]_{x=b}\right) \\
& \left.+\operatorname{ps}\left(\left[\mathrm{RL} J_{a}^{\alpha-3}(N u)\right]_{x=b}-\left[\mathrm{RL}_{a}^{\alpha-3}(g(x))\right]_{x=b}\right)\right] .
\end{aligned}
$$

Substituting (59) for $u^{\prime \prime}(a)$ and $u^{\prime \prime \prime}(a)$ into (54) and then solving the resulting equation for $u^{\prime}(a)$, we obtain the value of $u^{\prime}(a)$ as follows:

$$
\begin{aligned}
& u^{\prime}(a)=\frac{1}{b-a}\left[\sigma-\rho-\left[{ }_{\mathrm{RL}} J_{a}^{\alpha}(g(x))\right]_{x=b}\right. \\
& +\left[{ }_{\mathrm{RL}} J_{a}^{\alpha}(N u)\right]_{x=b}-\frac{(b-a)^{2}}{2 \Delta}(r \beta(b-a)+s \beta \\
& +q \gamma+q r\left(\left[\mathrm{RL} J_{a}^{\alpha-2}(N u)\right]_{x=b}\right. \\
& \left.\quad-\left[{ }_{\mathrm{RL}} J_{a}^{\alpha-2}(g(x))\right]_{x=b}\right)+q s\left(\left[\mathrm{RL}_{a}^{\alpha-3}(N u)\right]_{x=b}\right. \\
& \left.\left.\quad-\left[{ }_{\mathrm{RL}} J_{a}^{\alpha-3}(g(x))\right]_{x=b}\right)\right)+\frac{(b-a)^{3}}{6 \Delta}(r \beta-p \gamma \\
& +\operatorname{pr}\left(\left[\mathrm{RL}_{a} J_{a}^{\alpha-2}(g(x))\right]_{x=b}-\left[{ }_{\mathrm{RL}} J_{a}^{\alpha-2}(N u)\right]_{x=b}\right) \\
& +\operatorname{ps}\left(\left[\mathrm{RL} J_{a}^{\alpha-3}(g(x))\right]_{x=b}\right. \\
& \left.\left.\left.\quad-\left[{ }_{\mathrm{RL}} J_{a}^{\alpha-3}(N u)\right]_{x=b}\right)\right)\right] .
\end{aligned}
$$

For the computational convenience, we set

$$
\begin{aligned}
& \Delta=p s+q r+p r(b-a), \\
& \kappa=2 r \beta+p \gamma \\
& \lambda=s \beta+q \gamma \\
& \mu=2 b r \beta+b p \gamma \\
& \nu=p \gamma-r \beta \\
& \omega=3 q+b p-2 a p .
\end{aligned}
$$

Then we substitute (60) and (59) into (53) to obtain the following equivalent nonlinear Fredholm-Volterra integral equation:

$$
\begin{aligned}
& u(x)=\rho-\frac{(x-a)}{6(b-a) \Delta}\left(\kappa\left(b^{3}-a^{3}\right)+3 \lambda b^{2}\right. \\
& \left.+3(\lambda+\mu) a^{2}-3(2 \lambda+\mu) a b+6(\rho-\sigma) \Delta\right) \\
& +\frac{(x-a)^{2}}{2 \Delta}(r \beta(b-a)+\lambda)+\frac{(x-a)^{3}}{6 \Delta} v \\
& -\frac{(x-a)(x-b)(p x+\omega)}{6 \Delta}\left(s\left[{ }_{\mathrm{RL}} J_{a}^{\alpha-3}(g(x))\right]_{x=b}\right. \\
& \left.+r\left[{ }_{\mathrm{RL}} J_{a}^{\alpha-2}(g(x))\right]_{x=b}\right) \\
& -\frac{(x-a)}{(b-a)}\left[{ }_{\mathrm{RL}} J_{a}^{\alpha}(g(x))\right]_{x=b}+{ }_{\mathrm{RL}} J_{a}^{\alpha}(g(x)) \\
& +\frac{(x-a)(x-b)(p x+\omega)}{6 \Delta}\left(s\left[{ }_{\mathrm{RL}} J_{a}^{\alpha-3}(N u)\right]_{x=b}\right.
\end{aligned}
$$




$$
\begin{aligned}
& \left.+r\left[{ }_{\mathrm{RL}} J_{a}^{\alpha-2}(N u)\right]_{x=b}\right)+\frac{(x-a)}{(b-a)}\left[\mathrm{RL}_{a}^{\alpha}(N u)\right]_{x=b} \\
& -\mathrm{RL}_{a}^{\alpha} J_{a}^{\alpha}(N u),
\end{aligned}
$$

where ${ }_{\mathrm{RL}} J_{a}^{\alpha}(\cdot)$ is the Riemann-Liouville fractional integral operator of order $\alpha$ and where $\left.\left[{ }_{\mathrm{RL}} J_{a}^{\alpha}(\cdot)\right]_{x=b},{ }_{\mathrm{RL}} J_{a}^{\alpha-2}(\cdot)\right]_{x=b}$, and $\left[{ }_{\mathrm{RL}} J_{\mathrm{a}}^{\alpha-3}(\cdot)\right]_{x=b}$ are the operators of orders $\alpha, \alpha-2$, and $\alpha-3$ evaluated at $x=b$.

Substituting the equations in (37) into (62), we can determine the solution components from the following modified recursion scheme:

$$
\begin{aligned}
& u_{0}(x)=\rho-\frac{(x-a)}{6(b-a) \Delta}\left(\kappa\left(b^{3}-a^{3}\right)+3 \lambda b^{2}\right. \\
& \left.+3(\lambda+\mu) a^{2}-3(2 \lambda+\mu) a b+6(\rho-\sigma) \Delta\right) \\
& +\frac{(x-a)^{2}}{2 \Delta}(r \beta(b-a)+\lambda)+\frac{(x-a)^{3}}{6 \Delta} v \\
& -\frac{(x-a)(x-b)(p x+\omega)}{6 \Delta}\left(s\left[{ }_{\mathrm{RL}} J_{a}^{\alpha-3}(g(x))\right]_{x=b}\right. \\
& \left.+r\left[{ }_{\mathrm{RL}} J_{a}^{\alpha-2}(g(x))\right]_{x=b}\right) \\
& -\frac{(x-a)}{(b-a)}\left[{ }_{\mathrm{RL}} J_{a}^{\alpha}(g(x))\right]_{x=b}+{ }_{\mathrm{RL}} J_{a}^{\alpha}(g(x)), \\
& u_{n+1}(x) \\
& \quad=\frac{(x-a)(x-b)(p x+\omega)}{6 \Delta}\left(s\left[{ }_{\mathrm{RL}} J_{a}^{\alpha-3}\left(A_{n}\right)\right]_{x=b}\right. \\
& \left.+r\left[{ }_{\mathrm{RL}} J_{a}^{\alpha-2}\left(A_{n}\right)\right]_{x=b}\right)+\frac{(x-a)}{(b-a)}\left[{ }_{\mathrm{RL}} J_{a}^{\alpha}\left(A_{n}\right)\right]_{x=b} \\
& -\left[{ }_{\mathrm{RL}} J_{a}^{\alpha}\left(A_{n}\right)\right], \quad n \geq 0,
\end{aligned}
$$

where the resulting integrals are assumed to exist. The $n$-term approximation of the solution to the BVP can be obtained using (16). In addition, other techniques such as partitioning initial terms into two appropriate terms $[39,43,44]$ or using Duan's convergence parameter [40-42] can be incorporated, if necessary, into the recursion scheme (63) for solving the BVP described in (49)-(51).

Using the general formulas derived in (62) and (63), we can derive the equivalent nonlinear Fredholm-Volterra integral equations and their associated recursion schemes for (49) for the special cases of the boundary conditions in (50) and (51). The results are as follows.

Case 1. The nonlinear fractional BVP consists of (49) and the following two-point boundary conditions:

$$
\begin{gathered}
u(a)=\rho, \\
u^{\prime \prime}(a)=\beta, \\
u(b)=\sigma, \\
u^{\prime \prime}(b)=\gamma .
\end{gathered}
$$

The derivative boundary conditions (64) correspond to the case of $p=r=1$ and $q=s=0$ in (50) and (51). Thus (62) is then reduced to

$$
\begin{aligned}
& u(x)=\rho-\frac{(x-a)}{6(b-a) \Delta}\left(\kappa\left(b^{3}-a^{3}\right)+3 \mu a^{2}-3 \mu a b\right. \\
& +6(\rho-\sigma) \Delta)+\frac{\beta}{2}(x-a)^{2}+\frac{(x-a)^{3}}{6 \Delta} v \\
& -\frac{(x-a)(x-b)(x+\omega)}{6 \Delta}\left[{ }_{\mathrm{RL}} J_{a}^{\alpha-2}(g(x))\right]_{x=b} \\
& -\frac{(x-a)}{(b-a)}\left[{ }_{\mathrm{RL}} J_{a}^{\alpha}(g(x))\right]_{x=b}+{ }_{\mathrm{RL}} J_{a}^{\alpha}(g(x)) \\
& +\frac{(x-a)(x-b)(x+\omega)}{6 \Delta}\left[{ }_{\mathrm{RL}} J_{a}^{\alpha-2}(N u)\right]_{x=b} \\
& +\frac{(x-a)}{(b-a)}\left[{ }_{\mathrm{RL}} J_{a}^{\alpha}(N u)\right]_{x=b}-{ }_{\mathrm{RL}} J_{a}^{\alpha}(N u),
\end{aligned}
$$

where $\Delta=b-a \neq 0, \kappa=2 \beta+\gamma, \lambda=0, \mu=2 b \beta+b \gamma, \nu=\gamma-\beta$, and $\omega=b-2 a$ and where ${ }_{\mathrm{RL}} J_{a}^{\alpha}(\cdot)$ is the Riemann-Liouville fractional integral operator of order $\alpha$ and $\left.{ }_{\mathrm{RL}} J_{a}^{\alpha}(\cdot)\right]_{x=b}$ and $\left.{ }_{\mathrm{RL}} J_{a}^{\alpha-2}(\cdot)\right]_{x=b}$ are the operators of orders $\alpha$ and $\alpha-2$ evaluated at $x=b$.

Insertion of (37) into (65) gives the following modified recursion scheme for solution components:

$$
\begin{aligned}
& u_{0}(x)=\rho-\frac{(x-a)}{6(b-a) \Delta}\left(\kappa\left(b^{3}-a^{3}\right)+3 \mu a^{2}-3 \mu a b\right. \\
& +6(\rho-\sigma) \Delta)+\frac{\beta}{2}(x-a)^{2}+\frac{(x-a)^{3}}{6 \Delta} v \\
& -\frac{(x-a)(x-b)(x+\omega)}{6 \Delta}\left[{ }_{\mathrm{RL}} J_{a}^{\alpha-2}(g(x))\right]_{x=b} \\
& -\frac{(x-a)}{(b-a)}\left[{ }_{\mathrm{RL}} J_{a}^{\alpha}(g(x))\right]_{x=b}+{ }_{\mathrm{RL}} J_{a}^{\alpha}(g(x)), \\
& u_{n+1}(x)=\frac{(x-a)(x-b)(x+\omega)}{6 \Delta}\left[{ }_{\mathrm{RL}} J_{a}^{\alpha-2}\left(A_{n}\right)\right]_{x=b} \\
& +\frac{(x-a)}{(b-a)}\left[{ }_{\mathrm{RL}} J_{a}^{\alpha}\left(A_{n}\right)\right]_{x=b}-{ }_{\mathrm{RL}} J_{a}^{\alpha}\left(A_{n}\right), \quad n \geq 0,
\end{aligned}
$$

where we assume the resulting integrals exist.

Case 2. The nonlinear fractional BVP consists of (49) and the following two-point boundary conditions:

$$
\begin{aligned}
u(a) & =\rho, \\
-u^{\prime \prime \prime}(a) & =\beta, \\
u(b) & =\sigma, \\
u^{\prime \prime}(b) & =\gamma .
\end{aligned}
$$


The derivative boundary conditions (67) correspond to the case of $p=s=0$ and $q=r=1$ in (50) and (51). Thus we have $\Delta=1 \neq 0$ and $\omega=3$ and then (62) is reduced to

$$
\begin{aligned}
u(x) & =\rho-\frac{(x-a)}{6(b-a)}\left(\kappa\left(b^{3}-a^{3}\right)+3 \lambda b^{2}\right. \\
+ & \left.3(\lambda+\mu) a^{2}-3(2 \lambda+\mu) a b+6(\rho-\sigma)\right) \\
+ & \frac{(x-a)^{2}}{2}(\beta(b-a)+\lambda)+\frac{(x-a)^{3}}{6} v \\
- & \frac{(x-a)(x-b)}{2}\left[{ }_{\mathrm{RL}} J_{a}^{\alpha-2}(g(x))\right]_{x=b} \\
- & \frac{(x-a)}{(b-a)}\left[\mathrm{RL}_{a}^{\alpha}(g(x))\right]_{x=b}+{ }_{\mathrm{RL}} J_{a}^{\alpha}(g(x)) \\
+ & \frac{(x-a)(x-b)}{2}\left[{ }_{\mathrm{RL}} J_{a}^{\alpha-2}(\mathrm{Nu})\right]_{x=b} \\
+ & \frac{(x-a)}{(b-a)}\left[{ }_{\mathrm{RL}} J_{a}^{\alpha}(N u)\right]_{x=b}-{ }_{\mathrm{RL}} J_{a}^{\alpha}(N u),
\end{aligned}
$$

where $\kappa=2 \beta, \lambda=\gamma, \mu=2 b \beta$, and $\nu=-\beta$ and where ${ }_{\mathrm{RL}} J_{a}^{\alpha}(\cdot)$ is the Riemann-Liouville fractional integral operator of order $\alpha$ and where $\left[{ }_{\mathrm{RL}} J_{a}^{\alpha}(\cdot)\right]_{x=b}$ and $\left[{ }_{\mathrm{RL}} J_{a}^{\alpha-2}(\cdot)\right]_{x=b}$ are the operators of orders $\alpha$ and $\alpha-2$ evaluated at $x=b$.

Insertion of (37) into (68) gives the following modified recursion scheme for solution components:

$$
\begin{aligned}
& u_{0}(x)=\rho-\frac{(x-a)}{6(b-a)}\left(\kappa\left(b^{3}-a^{3}\right)+3 \lambda b^{2}\right. \\
& \left.+3(\lambda+\mu) a^{2}-3(2 \lambda+\mu) a b+6(\rho-\sigma)\right) \\
& +\frac{(x-a)^{2}}{2}(\beta(b-a)+\lambda)+\frac{(x-a)^{3}}{6} v \\
& -\frac{(x-a)(x-b)}{2}\left[\mathrm{RL}_{a}^{\alpha-2}(g(x))\right]_{x=b} \\
& -\frac{(x-a)}{(b-a)}\left[{ }_{\mathrm{RL}} J_{a}^{\alpha}(g(x))\right]_{x=b}+{ }_{\mathrm{RL}} J_{a}^{\alpha}(g(x)), \\
& u_{n+1}(x)=\frac{(x-a)(x-b)}{2}\left[{ }_{\mathrm{RL}} J_{a}^{\alpha-2}\left(A_{n}\right)\right]_{x=b} \\
& +\frac{(x-a)}{(b-a)}\left[{ }_{\mathrm{RL}} J_{a}^{\alpha}\left(A_{n}\right)\right]_{x=b}-{ }_{\mathrm{RL}} J_{a}^{\alpha}\left(A_{n}\right), \quad n \geq 0,
\end{aligned}
$$

where we assume the resulting integrals exist.

\section{Numerical Examples}

In this section, we demonstrate a use of the proposed recursion schemes in Section 3 derived from the DuanRach modified decomposition method to analytically and numerically solve nonlinear fractional BVPs. Several nonlinear fractional BVPs presented in this section correspond to the problems and their formulas described in Section 3 and some of these problems include physical and engineering problems such as problems of the Bratu type, a problem of the periodic base temperature in convective longitudinal fins, and an elastic beam problem. Numerical results obtained by the method are demonstrated graphically. Moreover, if the presented nonlinear fractional BVPs have their exact solutions then we will compute their corresponding maximal errors. Otherwise, we will investigate the error remainder function for the remaining problems.

In general, we consider a nonlinear fractional BVP: ${ }_{C} D_{a}^{\alpha} u(x)+N u=g(x), a \leq x \leq b$, where $\alpha$ is a fractional order of the equation imposed by some boundary conditions. If the exact solution $u^{*}(x)$ of the problem is known then we can examine the convergence of the $n$-term approximation $\varphi_{n}(x)=\sum_{k=0}^{n-1} u_{k}(x)$ from the error functions expressed as

$$
E_{n}(x)=\varphi_{n}(x)-u^{*}(x)
$$

and the maximal errors defined as

$$
\mathrm{ME}_{n}=\max _{a \leq x \leq b}\left|E_{n}(x)\right|
$$

For each value of $n$, we can calculate $\mathrm{ME}_{n}$ using the MATHEMATICA command "NMaximize" over an interval of interest. Then the logarithmic plot of these values of $\mathrm{ME}_{n}$ can be made using the MATHEMATICA command "ListLogPlot"; however, if the exact solution $u^{*}(x)$ of such a problem is unknown, we compute the error remainder functions defined as

$$
\begin{aligned}
\operatorname{ER}_{n}(x)={ }_{C} D_{a}^{\alpha} \varphi_{n}(x)+N \varphi_{n}(x)-g(x), & \\
& a \leq x \leq b .
\end{aligned}
$$

We observe that $\mathrm{ER}_{n}$ is the indicator for measuring how well the approximation $\varphi_{n}(x)$ satisfies the original nonlinear fractional differential equation.

Example 1. Consider the Bratu-type fractional BVP which is modified from the Bratu-type second-order equation in [12, $26,45,46]$ as follows:

$$
\begin{gathered}
{ }_{C} D_{0}^{\alpha} u+e^{u}=0, \quad 0 \leq x \leq 1, \quad 1<\alpha \leq 2, \\
u(0)=u(1)=0 .
\end{gathered}
$$

For $\alpha=2$, it can be easily verified that the exact solution of the BVP is

$$
\begin{aligned}
& u^{*}(x)=-2 \ln \frac{\cosh ((x-1 / 2)(C / 2))}{\cosh (C / 4)}, \\
& \text { where } C \text { satisfies } C=\sqrt{2} \cosh \left(\frac{C}{4}\right) .
\end{aligned}
$$

Fundamentally, we decompose the solution $u$ and the nonlinearity $N u=e^{u}$ as $u(x)=\sum_{n=0}^{\infty} u_{n}(x)$ and $N u=\sum_{n=0}^{\infty} A_{n}(x)$, where $u_{n}$ are the solution components and $A_{n}$ are the Adomian polynomials. We will show computational details for the proposed method, that is, the Duan-Rach modified 
ADM. We here list the first four Adomian polynomials for the nonlinear term $\mathrm{Nu}$ as follows:

$$
\begin{aligned}
& A_{0}=e^{u_{0}}, \\
& A_{1}=e^{u_{0}} u_{1}, \\
& A_{2}=\frac{1}{2} e^{u_{0}}\left(u_{1}^{2}+2 u_{2}\right), \\
& A_{3}=\frac{1}{6} e^{u_{0}}\left(u_{1}^{3}+6 u_{1} u_{2}+6 u_{3}\right) .
\end{aligned}
$$

The Duan-Rach Modified ADM. We can apply the formulas in (41) and (42) in Section 3.1 to this BVP. We have $a=0, b=1$, $p=1, q=0, r=1, s=0, \beta=0, \gamma=0$, and $\Delta=1$. Equation (41) turns out to be

$$
u(x)=x\left[{ }_{\mathrm{RL}} J_{0}^{\alpha}(N u)\right]_{x=1}-{ }_{\mathrm{RL}} J_{0}^{\alpha}(N u),
$$

where $N u=e^{u}$. Using (42) and Duan's convergence parameter technique, we obtain the following parametrized recursion scheme:

$$
\begin{aligned}
u_{0}(x) & =c, \\
u_{1}(x) & =-c+x\left[{ }_{\mathrm{RL}} J_{0}^{\alpha}\left(A_{0}\right)\right]_{x=1}-{ }_{\mathrm{RL}} J_{0}^{\alpha}\left(A_{0}\right), \\
u_{n+1}(x) & =x\left[{ }_{\mathrm{RL}} J_{0}^{\alpha}\left(A_{n}\right)\right]_{x=1}-{ }_{\mathrm{RL}} J_{0}^{\alpha}\left(A_{n}\right), \quad n \geq 1 .
\end{aligned}
$$

Since the expressions of the solution components $u_{4}(x)$, $u_{5}(x), u_{6}(x), \ldots$ are very long, we show only the solution components $u_{1}(x), u_{2}(x)$, and $u_{3}(x)$ using the Adomian polynomials $A_{0}, A_{1}$, and $A_{2}$ in (75) as follows:

$$
\begin{aligned}
& u_{1}(x)=-c+\frac{e^{c}}{\Gamma(\alpha+1)} x-\frac{e^{c}}{\Gamma(\alpha+1)} x^{\alpha}, \\
& u_{2}(x) \\
& \quad=\frac{4^{-\alpha} e^{c}\left(4^{\alpha} \Gamma(\alpha+1 / 2)\left(e^{c}-c \Gamma(\alpha+2)\right)-\sqrt{\pi} e^{c} \Gamma(\alpha+2)\right)}{\alpha \Gamma(\alpha) \Gamma(\alpha+1 / 2) \Gamma(\alpha+2)} \\
& \cdot x+\frac{c e^{c}}{\Gamma(\alpha+1)} x^{\alpha}-\frac{e^{2 c}}{\Gamma(\alpha+1) \Gamma(\alpha+2)} x^{\alpha+1}+\frac{e^{2 c}}{\Gamma(2 \alpha+1)} \\
& \cdot x^{2 \alpha}, \\
& u_{3}(x)=\frac{e^{c}}{2}\left(2 e ^ { c } \left(\frac{2 c}{\Gamma(2 \alpha+1)}\right.\right. \\
& +\frac{(\alpha+1)\left((2 \alpha+3) e^{c}-2 c \Gamma(\alpha+3)\right)}{\Gamma^{2}(\alpha+2) \Gamma(\alpha+3)} \\
& \left.\quad+\frac{(\alpha(\alpha+5)+3) e^{c}}{\Gamma(\alpha+2) \Gamma(2 \alpha+2)}+\frac{e^{c}}{\Gamma(3 \alpha+1)}\right)+\frac{c^{2}}{\Gamma(\alpha+1)} \\
& \left.+\frac{4^{\alpha} e^{2 c} \Gamma(\alpha+1 / 2)}{\sqrt{\pi} \Gamma(3 \alpha+1) \Gamma(\alpha+1)}\right) x-\frac{c^{2} e^{c}}{2 \Gamma(\alpha+1)} x^{\alpha}
\end{aligned}
$$

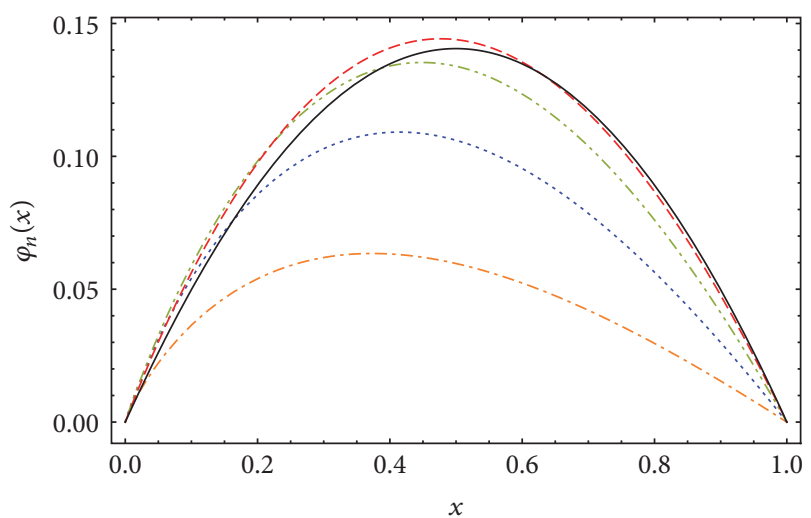

FIGURE 1: $\varphi_{n}(x)$ when $n=10$ obtained by the Duan-Rach modified ADM for $\alpha=1.2$ (dot-dash), $\alpha=1.4$ (dot line), $\alpha=1.6$ (dot-dotdash), $\alpha=1.8$ (dash line), and $\alpha=2$ (solid line).

$$
\begin{aligned}
& +\frac{e^{2 c}}{\Gamma^{3}(\alpha+2)}\left(-(\alpha+1) e^{c}+\frac{e^{c} \Gamma^{2}(\alpha+2)}{\Gamma(2 \alpha+1)}+2(\alpha+1) c \Gamma(\alpha\right. \\
& +2)) x^{\alpha+1}-\frac{e^{3 c}}{\Gamma^{2}(\alpha+1) \Gamma(\alpha+3)} x^{\alpha+2}-\frac{2 c e^{2 c}}{\Gamma(2 \alpha+1)} x^{2 \alpha} \\
& +\frac{(\alpha+2) e^{3 c}}{\Gamma(\alpha+1) \Gamma(2 \alpha+2)} x^{2 \alpha+1} \\
& -\frac{1}{2 \Gamma(\alpha+1) \Gamma(3 \alpha+1)}\left(2 \alpha e^{3 c} \Gamma(\alpha)+\frac{4^{\alpha} e^{3 c} \Gamma(\alpha+1 / 2)}{\sqrt{\pi}}\right) \\
& \cdot x^{3 \alpha} .
\end{aligned}
$$

Here we select $c=0.1$ for the above recursion scheme for the following values of $\alpha=1.2,1.4,1.6,1.8,2$. We can compute the approximate solution $\varphi_{n}(x)=\sum_{k=0}^{n-1} u_{k}(x)$ from the solution components $u_{0}(x), u_{1}(x), u_{2}(x), \ldots, u_{n-1}(x)$. In particular, we compute $\varphi_{10}(x)$ for each selected value of $\alpha$. Figure 1 shows curves of the approximation solutions $\varphi_{10}(x)$ for $\alpha=1.2,1.4,1.6,1.8,2$.

For each specified value of $\alpha \neq 2$, since the exact solution to this BVP is not known, we calculate the corresponding error remainder function $\mathrm{ER}_{n}(x)$ for $n=10$. Figure 2 displays the graphs of $\mathrm{ER}_{10}(x)$ for the specified values of $\alpha$. We can deduce from the graphs in Figure 2 that the approximations $\varphi_{10}(x)$ obtained by this method give remarkable accuracy, as expected, since when $n$ is sufficiently large the magnitude of each function $\operatorname{ER}_{n}(x)$ approaches zero.

For $\alpha=2$, it is possible to compute both the error function $E_{n}(x)$ and the maximal errors $\mathrm{ME}_{n}$; however, we only compute the values of $\mathrm{ME}_{n}$ for $n=2,3, \ldots, 9$ listed in Table 1. We show the logarithmic plots of $\mathrm{ME}_{n}$ versus $n$ for $n=2,3, \ldots, 9$ in Figure 3. We can observe in Figure 3 that all of the data points after $n=2$ lie almost on a straight line which demonstrates that the maximal errors $\mathrm{ME}_{n}$ are reduced approximately at an exponential rate.

Example 2. Consider the following nonlinear fractional order BVP which is developed from the second-order 


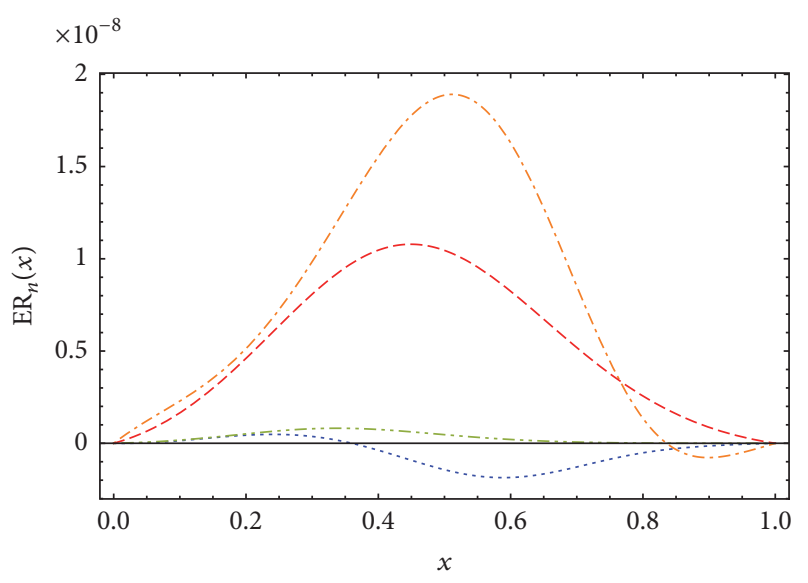

FIGURE 2: $\operatorname{ER}_{n}(x)$ when $n=10$ obtained by the Duan-Rach modified ADM for $\alpha=1.2$ (dot-dash), $\alpha=1.4$ (dot line), $\alpha=1.6$ (dot-dotdash), and $\alpha=1.8$ (dash line).

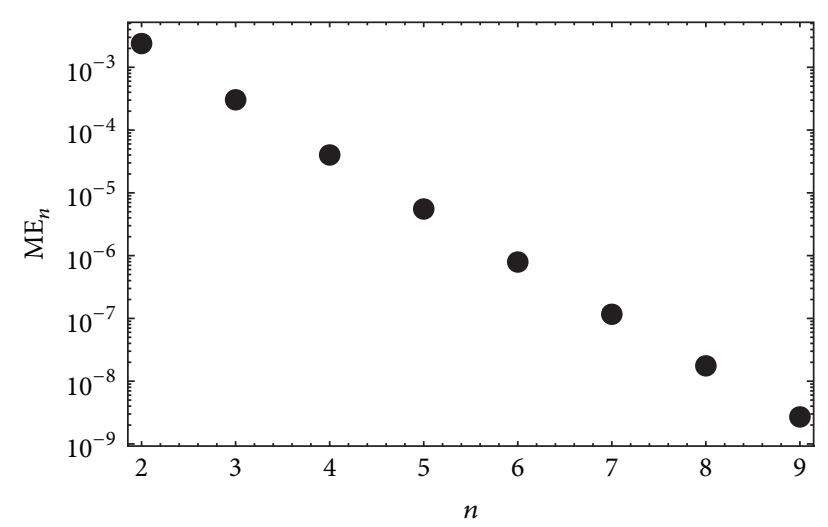

FIGURE 3: Logarithmic plots of the maximal errors $\mathrm{ME}_{n}$ versus $n$ for $n=2$ through 9 obtained by the Duan-Rach modified ADM.

homogeneous partial differential equation of the engineering model in [47] as follows:

$$
\begin{aligned}
& \frac{\partial^{\alpha} u(x, t)}{\partial x^{\alpha}}+\frac{\varepsilon}{1+\varepsilon u(x, t)}\left(\frac{\partial u(x, t)}{\partial x}\right)^{2} \\
& \quad-K^{2} \frac{u(x, t)}{1+\varepsilon u(x, t)}-\left(\frac{1}{1+\varepsilon u(x, t)}\right) \frac{\partial u(x, t)}{\partial t} \\
& \quad=0
\end{aligned}
$$

where the notation $\partial^{\alpha} / \partial x^{\alpha}$ in (79) represents a fractional partial derivative with respect to the space $x$ in the Caputo sense with the fractional order $\alpha \in(1,2]$ and where $u(x, t)$ has the domain of definition $x \in[0,1]$ and $t \in[0, \infty)$. The physical variables $u, x, t$ are the dimensionless temperature, distance, and time, respectively. For (79), the following mixed set of homogeneous Neumann and inhomogeneous Dirichlet boundary conditions is given as

$$
\begin{aligned}
& u_{x}(0, t)=0 \\
& u(1, t)=1+S \cos (B t)
\end{aligned}
$$

TABLE 1: For $c=0.1$ and $\alpha=2$, maximal errors $\mathrm{ME}_{n}$ for $n=2,3$, $\ldots, 9$ obtained by the Duan-Rach modified ADM.

\begin{tabular}{lc}
\hline$n$ & $\mathrm{ME}_{n}$ \\
\hline 2 & 0.00239285 \\
3 & 0.000303804 \\
4 & 0.0000401907 \\
5 & $5.53739 \times 10^{-6}$ \\
6 & $7.91736 \times 10^{-7}$ \\
7 & $1.16592 \times 10^{-7}$ \\
8 & $1.75801 \times 10^{-8}$ \\
9 & $2.70207 \times 10^{-9}$ \\
\hline
\end{tabular}

The above conditions consist of a sinusoidally varying boundary value. The parameters $\varepsilon, K, S$, and $B$ in (79) and (80) represent thermal conductivity parameter, fin parameter, amplitude of oscillation, and frequency of oscillation, respectively. The BVP in (79) and (80) describes physically the periodic base temperature in convective longitudinal fins.

The BVP in (79) and (80) has the same form as the BVP defined in (25) and (43) in Section 3.1. Thus, we have the nonlinearity $\mathrm{Nu}$ as follows:

$$
\begin{aligned}
N u= & \frac{\varepsilon}{1+\varepsilon u(x, t)}\left(u_{x}(x, t)\right)^{2}-K^{2} \frac{u(x, t)}{1+\varepsilon u(x, t)} \\
& -\frac{u_{t}(x, t)}{1+\varepsilon u(x, t)}
\end{aligned}
$$

and the source term $g(x, t)=0$. We decompose the solution $u$ and the nonlinearity $N u$ as $u(x, t)=\sum_{n=0}^{\infty} u_{n}(x, t)$ and $N u(x, t)=\sum_{n=0}^{\infty} A_{n}(x, t)$, where $u_{n}$ are the solution components and $A_{n}$ are the Adomian polynomials. Using (81), we list the first two Adomian polynomials for this nonlinear term as follows:

$$
\begin{aligned}
A_{0}= & -\frac{K^{2} u_{0}}{\varepsilon u_{0}+1}+\frac{\varepsilon\left(u_{0}^{(1,0)}\right)^{2}}{\varepsilon u_{0}+1}-\frac{u_{0}{ }^{(0,1)}}{\varepsilon u_{0}+1}, \\
A_{1}= & \frac{\varepsilon K^{2} u_{0} u_{1}}{\left(\varepsilon u_{0}+1\right)^{2}}-\frac{K^{2} u_{1}}{\varepsilon u_{0}+1}-\frac{\varepsilon^{2} u_{1}\left(u_{0}{ }^{(1,0)}\right)^{2}}{\left(\varepsilon u_{0}+1\right)^{2}} \\
& +\frac{\varepsilon u_{1} u_{0}{ }^{(0,1)}}{\left(\varepsilon u_{0}+1\right)^{2}}+\frac{2 \varepsilon u_{0}{ }^{(1,0)} u_{1}{ }^{(1,0)}}{\varepsilon u_{0}+1}-\frac{u_{1}{ }^{(0,1)}}{\varepsilon u_{0}+1},
\end{aligned}
$$

where the superscript $(1,0)$ denotes the differentiation with respect to $x$, while the superscript $(0,1)$ denotes the differentiation with respect to $t$ and so forth.

We employ the Duan-Rach modified ADM for the above BVP to which (44)-(45) are applied. Comparing with (43), we have $a=0, b=1, \beta=0, \gamma=1+S \cos (B t), p=s=0$, $q=r=1$, and $\Delta=-1$. Then (44) becomes

$$
\begin{aligned}
u(x, t)= & 1+S \cos (B t)+\left[{ }_{\mathrm{RL}} J_{0}^{\alpha}(N u)\right]_{x=1} \\
& -{ }_{\mathrm{RL}} J_{0}^{\alpha}(N u)
\end{aligned}
$$


Using (45), we obtain the following recursion scheme:

$$
\begin{aligned}
u_{0}(x, t) & =1+S \cos (B t), \\
u_{n+1}(x, t) & =\left[{ }_{\mathrm{RL}} J_{0}^{\alpha}\left(A_{n}\right)\right]_{x=1}-{ }_{\mathrm{RL}} J_{0}^{\alpha}\left(A_{n}\right) .
\end{aligned}
$$

Since the expressions of the solution components $u_{3}(x)$, $u_{4}(x), u_{5}(x), \ldots$ are complicated, we show only the solution components $u_{0}(x), u_{1}(x)$, and $u_{2}(x)$ using the Adomian polynomials $A_{0}$ and $A_{1}$ in (82) as follows:

$$
\begin{aligned}
& u_{0}(x, t)=1+S \cos (B t) \\
& \begin{aligned}
u_{1}(x, t) & =\frac{\left(x^{\alpha}-1\right)\left(K^{2} S \cos (B t)-B S \sin (B t)+K^{2}\right)}{\Gamma(\alpha+1)(\varepsilon S \cos (B t)+\varepsilon+1)}, \\
u_{2}(x, t) & =\frac{\left(x^{\alpha}-1\right)\left(\sqrt{\pi} \Gamma(\alpha+1)\left(x^{\alpha}+1\right)-4^{\alpha} \Gamma(\alpha+1 / 2)\right)}{2 \sqrt{\pi} \Gamma(\alpha+1) \Gamma(2 \alpha+1)(\varepsilon S \cos (B t)+\varepsilon+1)^{3}}\left(-2 S\left(B^{2}(\varepsilon+1)-K^{4}\right) \cos (B t)-3 B^{2} \varepsilon S^{2}+2 K^{4}\right. \\
& \left.+B S\left(2 K^{2} \sin (B t)(\varepsilon S \cos (B t)+\varepsilon-2)+B \varepsilon S \cos (2 B t)\right)\right) .
\end{aligned}
\end{aligned}
$$

We can compute the approximate solution $\varphi_{n}(x, t)=$ $\sum_{k=0}^{n-1} u_{k}(x, t)$ from the solution components $u_{0}(x, t), u_{1}(x, t)$, $u_{2}(x, t), \ldots, u_{n-1}(x, t)$. Since the time consumed for computing $\varphi_{n}(x, t)$ is long, we choose to calculate the approximate solution $\varphi_{5}(x, t)$. Here we take $\varepsilon=0.2, K=0.5, S=0.1$, and $B=1$ which are used to plot the approximate solutions and the error surfaces for $0 \leq x \leq 1$ and $0 \leq t \leq 4 \pi$. The threedimensional approximate solutions $\varphi_{5}(x, t)$ for the values of $\alpha=1.2,1.4,1.6,1.8,2$ are plotted in Figures 4(a) and 4(b) with various orientations. Figures $4(\mathrm{c})-4(\mathrm{e})$ show the cross sections of the approximate solutions $\varphi_{5}(x, t)$ at $x=0.2$, $x=0.5$, and $x=0.8$, respectively, for $\alpha=1.2,1.4,1.6,1.8,2$.

Since we do not yet know what the exact solution is for this BVP for each specified value of $\alpha$, the corresponding error remainder function

$$
\begin{aligned}
\operatorname{ER}_{n}(x, t)= & \frac{\partial^{\alpha} \varphi_{n}(x, t)}{\partial x^{\alpha}} \\
& +\frac{\varepsilon}{1+\varepsilon \varphi_{n}(x, t)}\left(\frac{\partial \varphi_{n}(x, t)}{\partial x}\right)^{2} \\
& -K^{2} \frac{\varphi_{n}(x, t)}{1+\varepsilon \varphi_{n}(x, t)} \\
& -\left(\frac{1}{1+\varepsilon \varphi_{n}(x, t)}\right) \frac{\partial \varphi_{n}(x, t)}{\partial t}
\end{aligned}
$$

is computed. Here we choose to compute $\mathrm{ER}_{n}(x, t)$ for only $\alpha=1.8$ by varying $n=2,3,4,5$. Figures $5(\mathrm{a})-5(\mathrm{~d})$ display the surfaces of the absolute error remainder functions $\left|\mathrm{ER}_{n}(x, t)\right|$ for $n=2,3,4,5$. We can deduce from Figures 5(a)-5(d) that for $\alpha=1.8$ the approximations $\varphi_{n}(x, t)$ obtained by this method provide the greater accuracy for larger $n$; that is, the maximum values of $\left|\mathrm{ER}_{n}(x, t)\right|$ decrease significantly as $n$ increases.

Example 3. The following nonlinear fractional BVP is modified from the nonlinear second-order BVP in [27] with the sum of an exponential nonlinearity in the solution and a quadratic nonlinearity in the derivative of the solution and subject to a set of Robin boundary conditions. The modified BVP can be expressed as follows:

$$
\begin{aligned}
{ }_{C} D_{0}^{\alpha} u(x)=-\frac{1}{8}\left(e^{-2 u}+4\left(u^{\prime}\right)^{2}\right), & \\
& 0 \leq x \leq 1,1<\alpha \leq 2,
\end{aligned}
$$

subject to the following set of Robin boundary conditions:

$$
\begin{aligned}
& u(0)-2 u^{\prime}(0)=-1, \\
& u(1)+2 u^{\prime}(1)=\frac{2}{3}+\ln \left(\frac{3}{2}\right) .
\end{aligned}
$$

Here we can rewrite (87) as

$$
{ }_{C} D_{0}^{\alpha} u(x)+\frac{1}{8}\left(e^{-2 u}+4\left(u^{\prime}\right)^{2}\right)=0,
$$

with the nonlinearity and the source term as follows:

$$
\begin{aligned}
N u & =\frac{1}{8}\left(e^{-2 u}+4\left(u^{\prime}\right)^{2}\right), \\
g(x) & =0 .
\end{aligned}
$$

For $\alpha=2$, it can be easily verified that the exact solution of the BVP is $u^{*}(x)=\ln ((2+x) / 2)$; however, the exact solution to this BVP is not known for $1<\alpha<2$. Next we decompose the solution $u$ and the nonlinearity $N u$ as $u(x)=\sum_{n=0}^{\infty} u_{n}(x)$ and $N u=\sum_{n=0}^{\infty} A_{n}(x)$, where $u_{n}$ are the solution components and $A_{n}$ are the Adomian polynomials. We here list the first four Adomian polynomials for the nonlinear term as follows:

$$
\begin{aligned}
A_{0} & =\frac{1}{8}\left(e^{-2 u_{0}}+4\left(u_{0}^{\prime}\right)^{2}\right), \\
A_{1} & =-\frac{1}{4} e^{-2 u_{0}} u_{1}+u_{0}^{\prime} u_{1}^{\prime}, \\
A_{2} & =\frac{1}{4} e^{-2 u_{0}}\left(u_{1}^{2}-u_{2}+2 e^{2 u_{0}}\left(\left(u_{1}^{\prime}\right)^{2}+2 u_{0}^{\prime} u_{2}^{\prime}\right)\right), \\
A_{3} & =\frac{1}{12} e^{-2 u_{0}}\left(-2 u_{1}^{3}+6 u_{1} u_{2}\right. \\
& \left.-3\left(u_{3}-4 e^{2 u_{0}}\left(u_{1}^{\prime} u_{2}^{\prime}+u_{0}^{\prime} u_{3}^{\prime}\right)\right)\right) .
\end{aligned}
$$



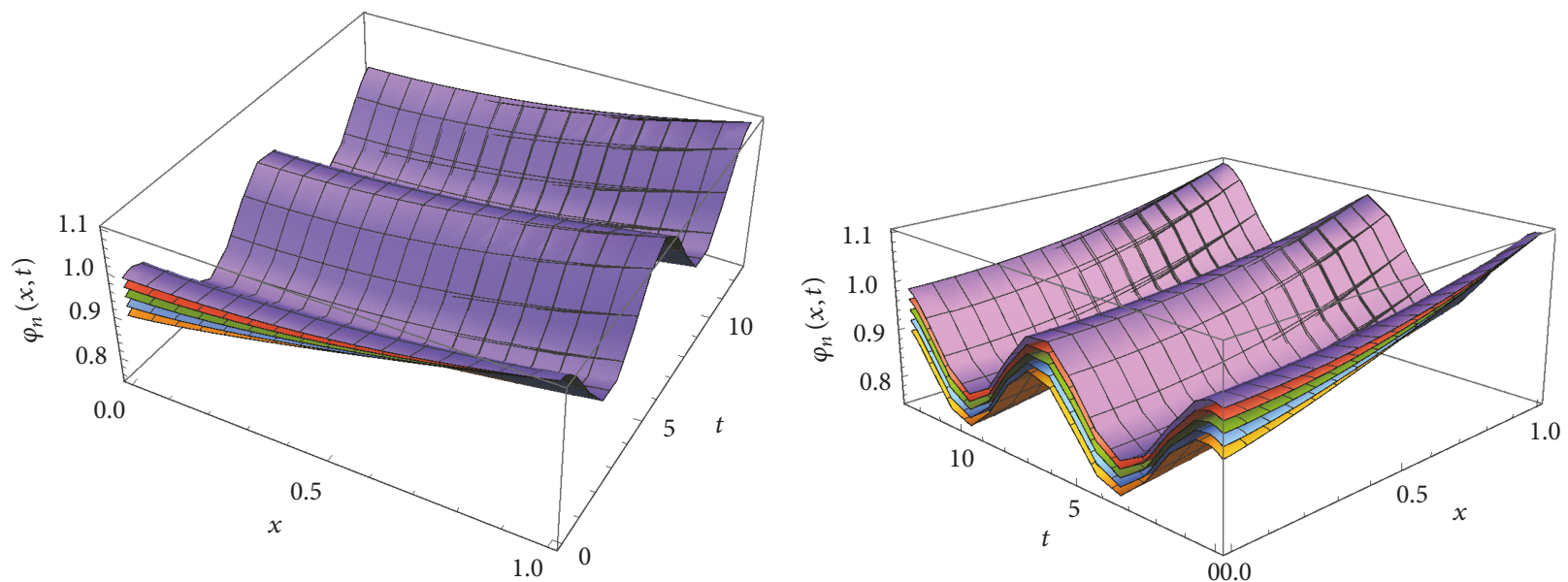

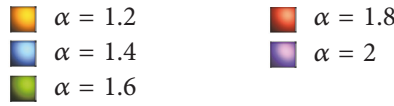

(a)

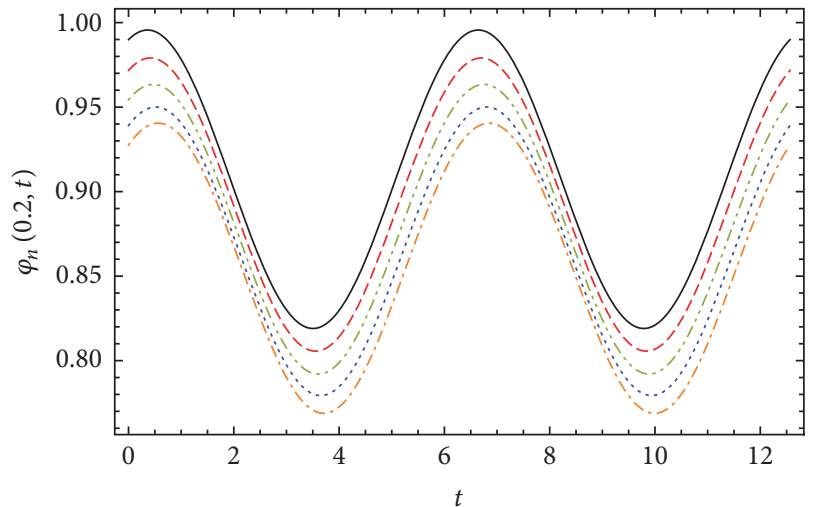

$\begin{aligned} \cdots-\alpha & =1.2 \\ \cdots-\alpha & =1.4 \\ \cdots & \alpha=1.6\end{aligned}$ $---\alpha=1.8$

$\alpha=2$ $\square \alpha=1.2$

$\alpha=1.4$

$\alpha=1.6$

$\alpha=1.8$

$\alpha=2$

(b)

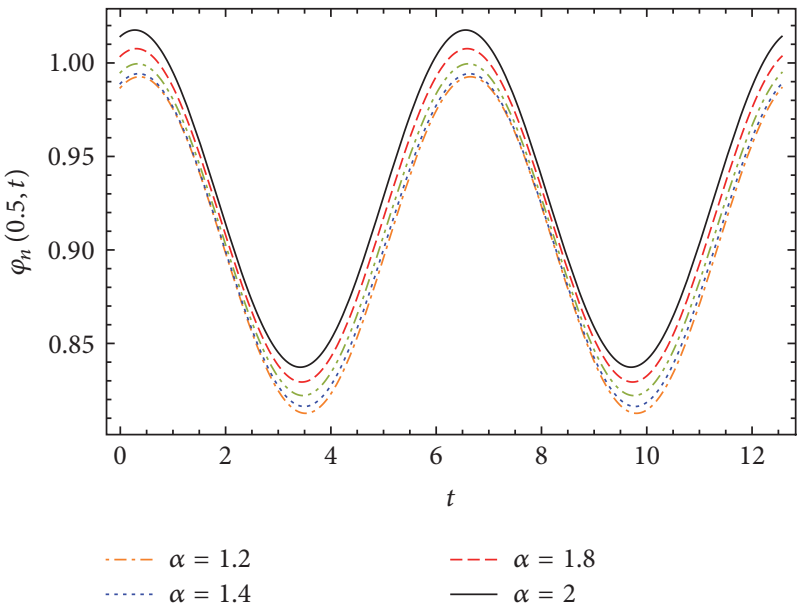

(d)

(c)

$\cdots \quad \alpha=1.6$

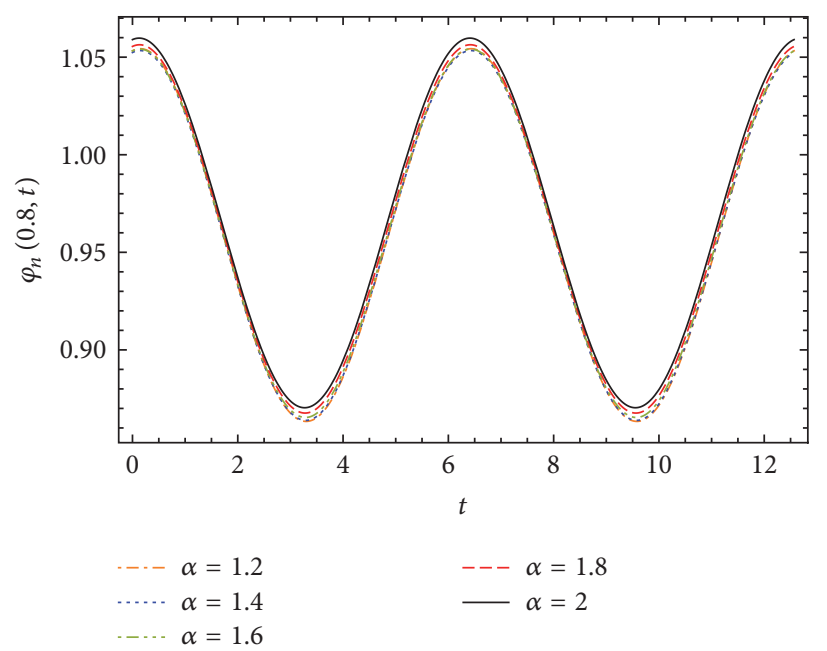

(e)

FIGURE 4: ((a)-(b)) Surfaces of approximate solutions $\varphi_{5}(x, t)$ for the values of $\alpha=1.2,1.4,1.6,1.8,2$ with different angles of view. ((c)-(e)) Cross sections of the surfaces of $\varphi_{5}(x, t)$ at $x=0.2, x=0.5$, and $x=0.8$, respectively, for $\alpha=1.2$ (dot-dash), $\alpha=1.4$ (dot line), $\alpha=1.6$ (dot-dot-dash), $\alpha=1.8$ (dash line), and $\alpha=2$ (solid line). 
The Duan-Rach Modified ADM. We can now apply the formulas in (36) and (38) in Section 3.1 to this BVP. For the parameters described in such a section, we have $a=0, b=1$, $p=1, q=-2, r=1, s=2, \beta=-1, \gamma=2 / 3+\ln (3 / 2)$, and $\Delta=5$. Equation (36) turns out to be

$$
\begin{aligned}
& u(x)=\frac{1}{5}\left[\left(-\frac{5}{3}+2 \ln \left(\frac{3}{2}\right)\right)+\left(\frac{5}{3}+\ln \left(\frac{3}{2}\right)\right) x\right. \\
& +(2 x+4)\left[{ }_{\mathrm{RL}} J_{0}^{\alpha-1}(N u)\right]_{x=1} \\
& \left.+(x+2)\left[{ }_{\mathrm{RL}} J_{0}^{\alpha}(N u)\right]_{x=1}\right]-{ }_{\mathrm{RL}} J_{0}^{\alpha}(N u) .
\end{aligned}
$$

Using (38) and Duan's convergence parameter technique, we obtain the following parametrized recursion scheme:

$$
\begin{aligned}
& u_{0}(x)=c, \\
& u_{1}(x)=-c+\frac{1}{5}\left(-\frac{5}{3}+2 \ln \left(\frac{3}{2}\right)\right)
\end{aligned}
$$

$$
\begin{aligned}
+ & \frac{1}{5}\left(\frac{5}{3}+\ln \left(\frac{3}{2}\right)\right) x \\
+ & \frac{(2 x+4)}{5}\left[{ }_{\mathrm{RL}} J_{0}^{\alpha-1}\left(A_{0}\right)\right]_{x=1} \\
& +\frac{(x+2)}{5}\left[{ }_{\mathrm{RL}} J_{0}^{\alpha}\left(A_{0}\right)\right]_{x=1} \\
& -\mathrm{RL}_{0}^{\alpha}\left(A_{0}\right), \\
u_{n+1}(x)= & \frac{(2 x+4)}{5}\left[{ }_{\mathrm{RL}} J_{0}^{\alpha-1}\left(A_{n}\right)\right]_{x=1} \\
& +\frac{(x+2)}{5}\left[{ }_{\mathrm{RL}} J_{0}^{\alpha}\left(A_{n}\right)\right]_{x=1} \\
& -{ }_{\mathrm{RL}} J_{0}^{\alpha}\left(A_{n}\right), \quad n \geq 1 .
\end{aligned}
$$

Since the expressions of the solution components $u_{n}(x)$, $n \geq 2$, are quite long, we show only the first two solution components $u_{1}(x)$ and $u_{2}(x)$ computed using the Adomian polynomials $A_{0}$ and $A_{1}$ in (91) as follows:

$$
\begin{aligned}
& u_{1}(x)=-c-\frac{1}{3}+\frac{2}{5} \ln \left(\frac{3}{2}\right)+\frac{(2 \alpha+1) e^{-2 c}}{20 \Gamma(\alpha+1)}+\left(\frac{1}{3}+\frac{1}{5} \ln \left(\frac{3}{2}\right)+\frac{(2 \alpha+1) e^{-2 c}}{40 \Gamma(\alpha+1)}\right) x-\frac{e^{-2 c}}{8 \Gamma(\alpha+1)} x^{\alpha}, \\
& u_{2}(x)=\frac{e^{-4 c}}{1200}\left(\frac{60 \alpha+15}{\Gamma(2 \alpha+1)}-\frac{3(\alpha+1)(2 \alpha+1)(4 \alpha(\alpha+2)+5)}{\Gamma^{2}(\alpha+2)}\right. \\
& \left.+\frac{8 e^{2 c}\left(\alpha\left(2 \alpha(5-6 \ln (3)+\ln (64))+5-48 \operatorname{coth}^{-1}(5)\right)+15(\alpha+1)(2 \alpha+1) c-10\left(1+3 \operatorname{coth}^{-1}(5)\right)\right)}{\Gamma(\alpha+2)}\right) \\
& +\frac{e^{-4 c}}{2400}\left(\frac{60 \alpha+15}{\Gamma(2 \alpha+1)}-\frac{3(\alpha+1)(2 \alpha+1)(4 \alpha(\alpha+2)+5)}{\Gamma^{2}(\alpha+2)}\right. \\
& \left.+\frac{8 e^{2 c}\left(\alpha\left(2 \alpha(5-6 \ln (3)+\ln (64))+5-48 \operatorname{coth}^{-1}(5)\right)+15(\alpha+1)(2 \alpha+1) c-10\left(1+3 \operatorname{coth}^{-1}(5)\right)\right)}{\Gamma(\alpha+2)}\right) x \\
& +\frac{e^{-4 c}\left(6 \alpha^{2}+9 \alpha-4 e^{2 c} \Gamma(\alpha+2)(15 c+5-6 \ln (3)+\ln (64))+3\right)}{240 \alpha \Gamma(\alpha) \Gamma(\alpha+2)} x^{\alpha} \\
& +\frac{e^{-4 c}\left(3(\alpha+1)(2 \alpha+1)+8 e^{2 c}(5+\ln (27 / 8)) \Gamma(\alpha+2)\right)}{480 \Gamma^{2}(\alpha+2)} x^{\alpha+1}-\frac{e^{-4 c}}{32 \Gamma(2 \alpha+1)} x^{2 \alpha} .
\end{aligned}
$$

Throughout this method, we use $c=0.5$ and the following values of $\alpha=1.2,1.4,1.6,1.8,2$ for the recursion scheme in (93). We can compute the approximate solution $\varphi_{n}(x)=\sum_{k=0}^{n-1} u_{k}(x)$ from the solution components $u_{0}(x)$, $u_{1}(x), u_{2}(x), \ldots, u_{n-1}(x)$. In particular, we calculate $\varphi_{15}(x)$ for each selected value of $\alpha$ using the Duan-Rach modified ADM. Figure 6 shows curves of the approximate solutions $\varphi_{15}(x)$ for $\alpha=1.2,1.4,1.6,1.8,2$.

For each specified value of $\alpha \neq 2$, since the exact solution to this BVP is not known, we calculate the corresponding error remainder functions $\operatorname{ER}_{n}(x)$ for $n=15$. Figure 7 displays the graphs of $\mathrm{ER}_{15}(x)$ for the specified values of $\alpha$. We can deduce from the graphs in Figure 7 that the approximations $\varphi_{15}(x)$ obtained by this method give the remarkable accuracy as expected that when $n$ is sufficiently large then the magnitude of each function $\operatorname{ER}_{n}(x)$ approaches zero.

For $\alpha=2$, it is possible to compute both of the error functions $E_{n}(x)$ and the maximal errors $\mathrm{ME}_{n}$; however, we only compute the values of $\mathrm{ME}_{n}$ for $n=2,3, \ldots, 15$ listed in Table 2. We show the logarithmic plots of $\mathrm{ME}_{n}$ versus $n$ for $n=2,3, \ldots, 15$ in Figure 8. We can observe in Figure 8 


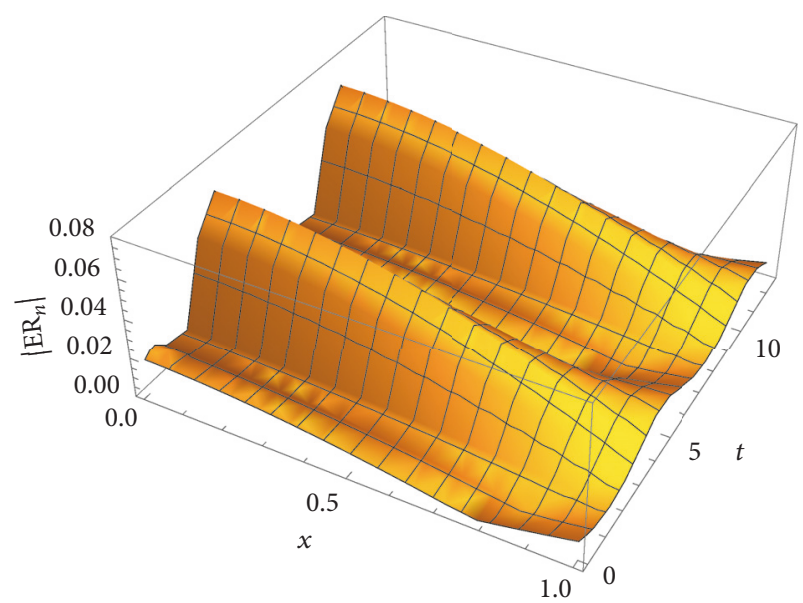

(a)

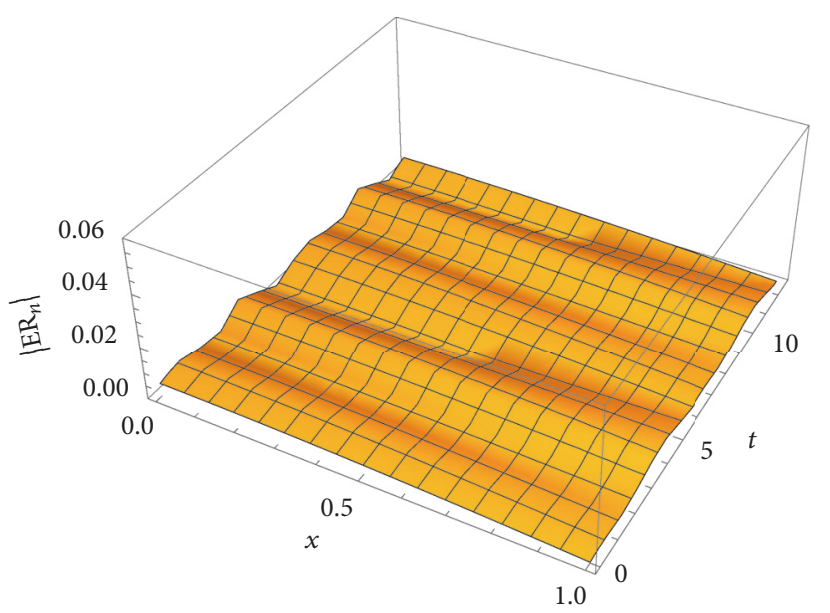

(c)

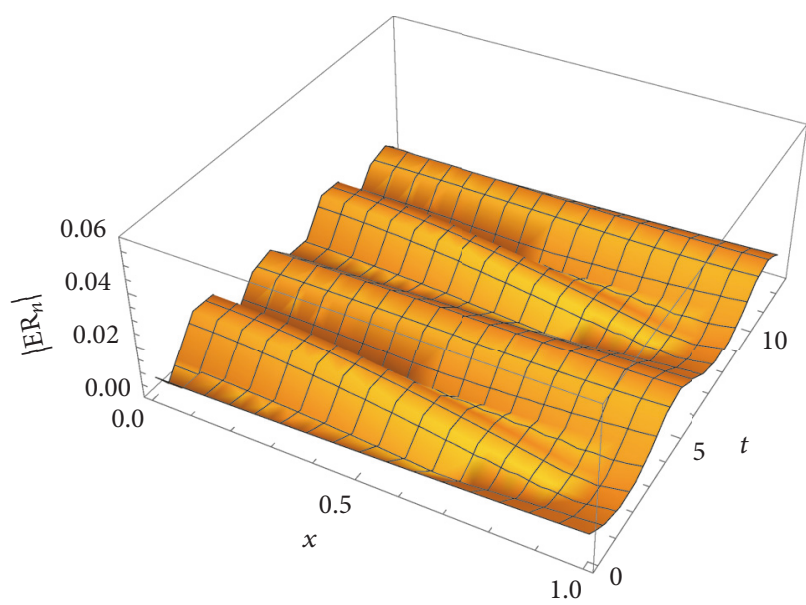

(b)

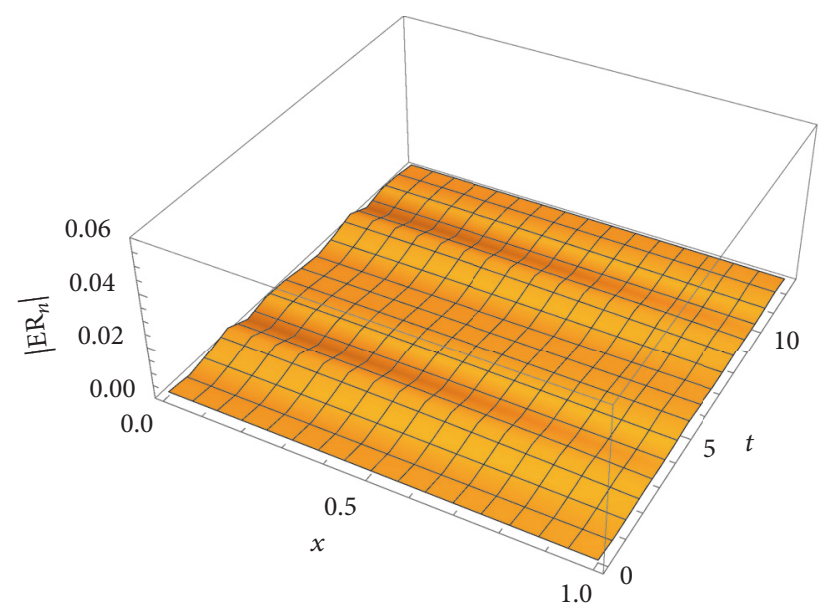

(d)

FIGURE 5: Surfaces of the absolute error remainder functions for $\alpha=1.8,(\mathrm{a})\left|\mathrm{ER}_{2}(x, t)\right|,(\mathrm{b})\left|\mathrm{ER}_{3}(x, t)\right|,(\mathrm{c})\left|\mathrm{ER}_{4}(x, t)\right|$, and $(\mathrm{d})\left|\mathrm{ER}_{5}(x, t)\right|$.

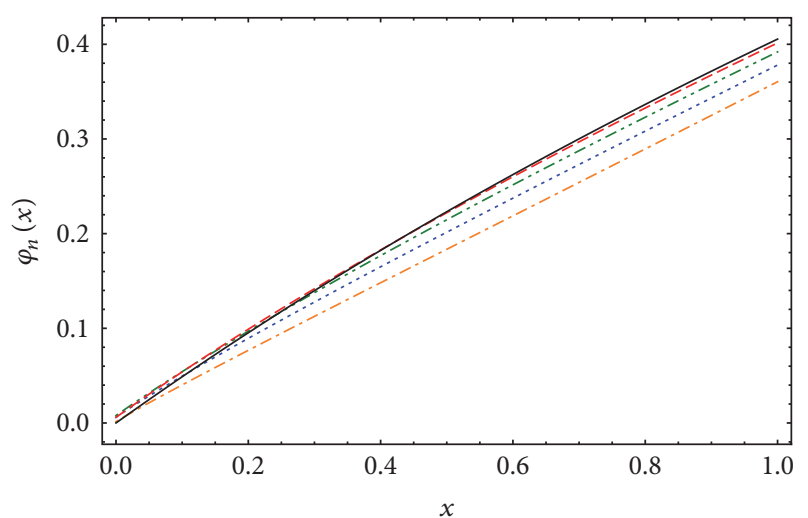

FIGURE 6: $\varphi_{n}(x)$ when $n=15$ obtained by the Duan-Rach modified ADM for $\alpha=1.2$ (dot-dash), $\alpha=1.4$ (dot line), $\alpha=1.6$ (dot-dotdash), $\alpha=1.8$ (dash line), and $\alpha=2$ (solid line).

that all of the data points after $n=7$ lie almost on a straight line which demonstrates the maximal errors $\mathrm{ME}_{n}$ are reduced approximately at an exponential rate.
Example 4. Consider the beam-type fractional boundary value problem

$$
\begin{aligned}
{ }_{C} D_{0}^{\alpha} u-2 u^{\prime \prime}+u & =g(x), \quad 0 \leq x \leq 1,3<\alpha \leq 4, \\
u(0) & =0, \\
u^{\prime \prime}(0) & =0, \\
u(1) & =0, \\
u^{\prime \prime}(1) & =-12,
\end{aligned}
$$

where $g(x)=-24+x+24 x^{2}-x^{4}$. For $\alpha=4$, it can be easily verified that the exact solution of the BVP is $u^{*}(x)=$ $x\left(1-x^{3}\right)$. Fundamentally, we decompose the solution $u$ and the nonlinearity $N u=u-2 u^{\prime \prime}$ as $u(x)=\sum_{n=0}^{\infty} u_{n}(x)$ and $N u=\sum_{n=0}^{\infty} A_{n}(x)$, where $u_{n}$ are the solution components and $A_{n}$ are the Adomian polynomials. We here list the first four Adomian polynomials for this nonlinear term as follows:

$$
\begin{aligned}
& A_{0}=u_{0}-2 u_{0}^{\prime \prime}, \\
& A_{1}=u_{1}-2 u_{1}^{\prime \prime},
\end{aligned}
$$




$$
\begin{aligned}
& A_{2}=u_{2}-2 u_{2}^{\prime \prime}, \\
& A_{3}=u_{3}-2 u_{3}^{\prime \prime} .
\end{aligned}
$$

The Duan-Rach Modified ADM. We can apply the formulas in (65) and (66) in Section 3.2 to this BVP. We have $a=0, b=1$, $p=1, q=0, r=1, s=0, \rho=0, \sigma=0, \beta=0, \gamma=-12$, and $\Delta=1$. Then, we have $\kappa=-12, \lambda=0, \mu=-12, \nu=-12$, and $\omega=1$. Thus, (65) turns out to be

$$
\begin{aligned}
u(x)= & 2 x-2 x^{3}-\frac{1}{6}\left(x^{3}-x\right)\left[{ }_{\mathrm{RL}} J_{0}^{\alpha-2}(g(x))\right]_{x=1} \\
& -x\left[{ }_{\mathrm{RL}} J_{0}^{\alpha}(g(x))\right]_{x=1}+{ }_{\mathrm{RL}} J_{0}^{\alpha}(g(x)) \\
& +\frac{1}{6}\left(x^{3}-x\right)\left[{ }_{\mathrm{RL}} J_{0}^{\alpha-2}(\mathrm{Nu})\right]_{x=1} \\
& +x\left[{ }_{\mathrm{RL}} J_{0}^{\alpha}(N u)\right]_{x=1}-{ }_{\mathrm{RL}} J_{0}^{\alpha}(N u)
\end{aligned}
$$

$$
\begin{aligned}
& u_{0}(x)=\left(2+\frac{-24 \alpha^{6}-215 \alpha^{5}-398 \alpha^{4}+1589 \alpha^{3}+7028 \alpha^{2}+7860 \alpha+864}{6 \Gamma(\alpha+5)}\right) x+(-2 \\
& \left.+\frac{24 \alpha^{4}+47 \alpha^{3}-75 \alpha^{2}-194 \alpha-72}{6 \Gamma(\alpha+3)}\right) x^{3}-\frac{24}{\Gamma(\alpha+1)} x^{\alpha}+\frac{x^{\alpha+1}}{\Gamma(\alpha+2)}+\frac{48}{\Gamma(\alpha+3)} x^{\alpha+2}-\frac{24}{\Gamma(\alpha+5)} x^{\alpha+4}, \\
& u_{1}(x)=\frac{1}{36}\left(\frac{432 / \Gamma(\alpha+3)+72}{\Gamma(\alpha+2)}-\frac{156 \alpha^{4}+936 \alpha^{3}+780 \alpha^{2}-3744 \alpha-5184}{\Gamma(\alpha+4)}\right. \\
& +\frac{312 \alpha^{9}+3731 \alpha^{8}+13895 \alpha^{7}+755 \alpha^{6}-111451 \alpha^{5}-216262 \alpha^{4}+64052 \alpha^{3}+576816 \alpha^{2}+544464 \alpha+124416}{\Gamma(\alpha+3) \Gamma(\alpha+5)} \\
& \left.-\frac{73728 \alpha^{8}+145920 \alpha^{7}-420096 \alpha^{6}-1221312 \alpha^{5}-655872 \alpha^{4}+576432 \alpha^{3}+897120 \alpha^{2}+474480 \alpha+74304}{\Gamma(2 \alpha+5)}\right) x \\
& +\left(\frac{4 \alpha^{4}+8 \alpha^{3}-12 \alpha^{2}-32 \alpha-2 \Gamma(\alpha+3)-12}{\Gamma(\alpha+2) \Gamma(\alpha+3)}+\frac{1536 \alpha^{6}-2336 \alpha^{5}-2880 \alpha^{4}+1964 \alpha^{3}+822 \alpha^{2}-54 \alpha+84}{3 \Gamma(2 \alpha+3)}\right. \\
& \left.+\frac{13}{36 \Gamma^{2}(\alpha)}+\frac{-26 \alpha^{6}-234 \alpha^{5}-586 \alpha^{4}+249 \alpha^{3}+2857 \alpha^{2}+3448 \alpha+13 \Gamma(\alpha+5)+924}{3 \Gamma(\alpha) \Gamma(\alpha+5)}\right) x^{3} \\
& +\frac{\left(312 \alpha^{6}+2795 \alpha^{5}+6902 \alpha^{4}-3449 \alpha^{3}-34988 \alpha^{2}-41844 \alpha-156 \Gamma(\alpha+5)-11232\right)}{6 \Gamma(\alpha+2) \Gamma(\alpha+5)} x^{\alpha+1} \\
& +\frac{\left(-24 \alpha^{4}-47 \alpha^{3}+75 \alpha^{2}+194 \alpha+12 \Gamma(\alpha+3)+72\right)}{\Gamma(\alpha+3) \Gamma(\alpha+4)} x^{\alpha+3}-\frac{48}{\Gamma(2 \alpha-1)} x^{2 \alpha-2}+\frac{2}{\Gamma(2 \alpha)} x^{2 \alpha-1}+\frac{120}{\Gamma(2 \alpha+1)} x^{2 \alpha} \\
& -\frac{x^{2 \alpha+1}}{\Gamma(2 \alpha+2)}-\frac{96}{\Gamma(2 \alpha+3)} x^{2 \alpha+2}+\frac{24}{\Gamma(2 \alpha+5)} x^{2 \alpha+4} \text {. }
\end{aligned}
$$

In particular, we calculate $\varphi_{10}(x)$ for $\alpha=3.2,3.4,3.6,3.8$, 4. Figure 9 shows curves of the approximate solutions $\varphi_{10}(x)$ for the selected values of $\alpha$.

For $\alpha=3.2,3.4,3.6,3.8$, we do not know what the exacts solutions are, and thus we calculate the error remainder functions $\operatorname{ER}_{n}(x)={ }_{C} D_{0}^{\alpha} \varphi_{n}(x)-2 \varphi_{n}^{\prime \prime}(x)+\varphi_{n}(x)-g(x)$,
$0 \leq x \leq 1$, for each value of $\alpha$. We display the functions $\operatorname{ER}_{n}(x)$ for $n=10$ in Figure 10. The approximations of this order give significant accuracy, as expected, since the limit of the functions $\operatorname{ER}_{n}(x)$ approaches zero.

For $\alpha=4$, it is possible to compute the error functions $E_{n}$ in (70) as $E_{n}=\varphi_{n}(x)-u^{*}(x)$ and the maximal errors $\mathrm{ME}_{n}$ in 


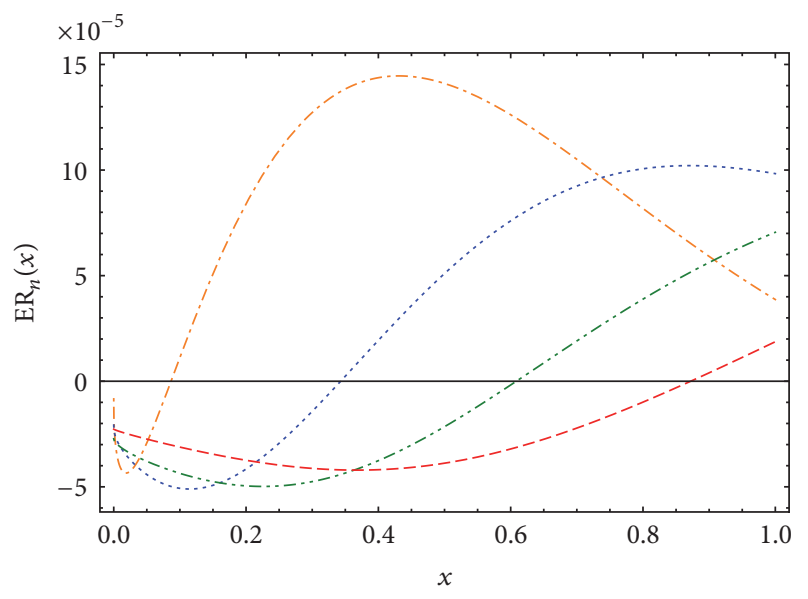

FIGURE 7: $\operatorname{ER}_{n}(x)$ when $n=15$ obtained by the Duan-Rach modified ADM for $\alpha=1.2$ (dot-dash), $\alpha=1.4$ (dot line), $\alpha=1.6$ (dot-dotdash), $\alpha=1.8$ (dash line), and $\alpha=2$ (solid line).

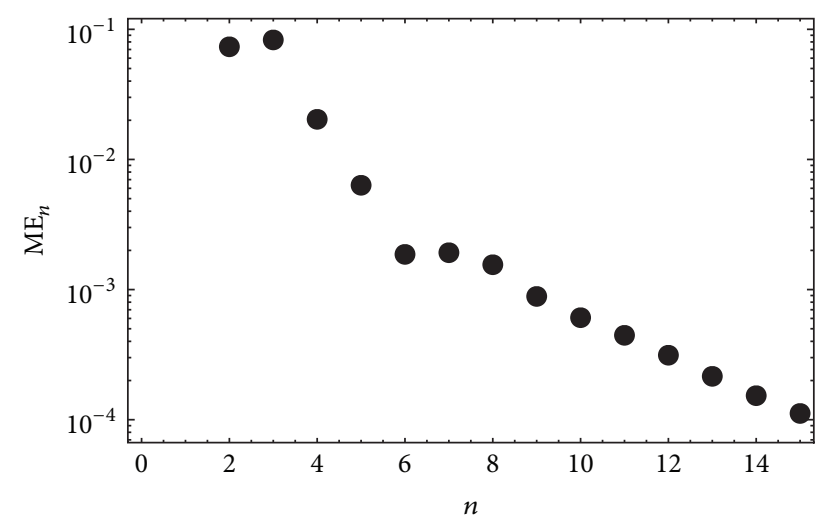

FIGURE 8: Logarithmic plots of the maximal errors $\mathrm{ME}_{n}$ versus $n$ for $n=2$ through 15 obtained by the Duan-Rach modified ADM.

(71) as $\mathrm{ME}_{n}=\max _{0 \leq x \leq 1}\left|E_{n}(x)\right|$. However, we only compute the values of $\mathrm{ME}_{n}$ for $n=2,3,4, \ldots, 9$ listed in Table 3 . In Figure 11 we show the logarithmic plots of $\mathrm{ME}_{n}$ versus $n$ for $n=2,3,4, \ldots, 9$ obtained by the method. All of the data points lie almost on a straight line so the maximal errors are decreasing approximately at an exponential rate.

\section{Conclusions}

We have established new recursion schemes using the DuanRach modified decomposition method to solve a variety of nonlinear fractional BVPs. The obtained recursion schemes have been derived for solving the nonlinear fractional BVPs with a set of Robin boundary conditions (order $1<\alpha \leq 2$ ) and with separated boundary conditions (order $3<\alpha \leq$ 4). We have applied the new recursion schemes to four numerical expository examples. Example 1 is a nonlinear Dirichlet fractional BVP of order $1<\alpha \leq 2$ with the exponential nonlinearity. Example 2 is a nonlinear Neumann and Dirichlet fractional BVP of order $1<\alpha \leq 2$ with the first derivative nonlinearity. Example 3 is a fractional BVP

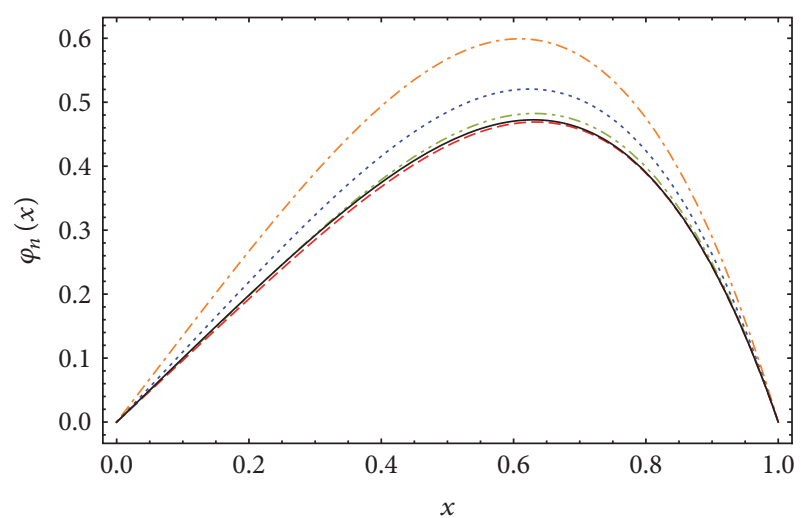

FIGURE 9: $\varphi_{n}(x)$ when $n=10$ obtained by the Duan-Rach modified ADM for $\alpha=3.2$ (dot-dash), $\alpha=3.4$ (dot line), $\alpha=3.6$ (dot-dotdash), $\alpha=3.8$ (dash line), and $\alpha=4$ (solid line).

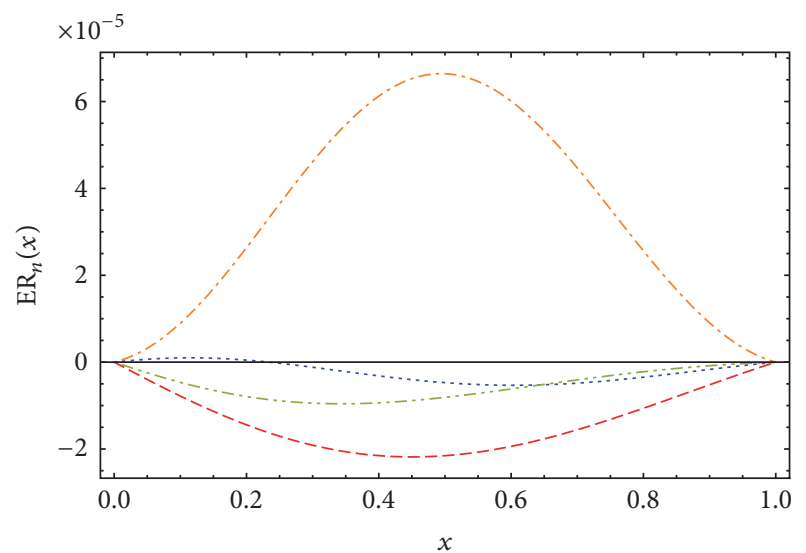

FIgURE 10: $\operatorname{ER}_{n}(x)$ when $n=10$ obtained by the Duan-Rach modified ADM for $\alpha=3.2$ (dot-dash), $\alpha=3.4$ (dot line), $\alpha=3.6$ (dot-dot-dash), and $\alpha=3.8$ (dash line).

TABLE 2: For $c=0.5$ and $\alpha=2$, maximal errors $\mathrm{ME}_{n}$ for $n=$ $2,3, \ldots, 15$ obtained by the Duan-Rach modified ADM.

\begin{tabular}{lc}
\hline$n$ & $\mathrm{ME}_{n}$ \\
\hline 2 & 0.0734942 \\
3 & 0.0830039 \\
4 & 0.0203679 \\
5 & 0.00633962 \\
6 & 0.00186625 \\
7 & 0.00192242 \\
8 & 0.00155408 \\
9 & 0.000886808 \\
10 & 0.000608945 \\
11 & 0.000445665 \\
12 & 0.000313388 \\
13 & 0.00021568 \\
14 & 0.00015314 \\
15 & 0.000111835 \\
\hline
\end{tabular}

with the sum of an exponential nonlinearity in the solution and a quadratic nonlinearity in the derivative of the solution 


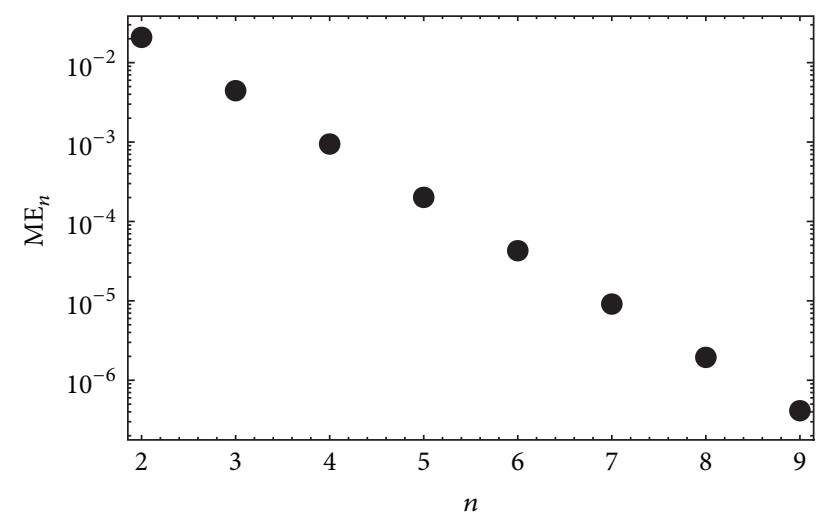

FIGURE 11: Logarithmic plots of the maximal errors $\mathrm{ME}_{n}$ versus $n$ for $n=2$ through 9 obtained by the Duan-Rach modified ADM.

TABLE 3: For $\alpha=4$, maximal errors $\mathrm{ME}_{n}$ for $n=2,3, \ldots, 9$ obtained by the Duan-Rach modified ADM.

\begin{tabular}{lc}
\hline$n$ & $\mathrm{ME}_{n}$ \\
\hline 2 & 0.0208592 \\
3 & 0.00444248 \\
4 & 0.000945886 \\
5 & 0.000201388 \\
6 & 0.0000428772 \\
7 & $9.12892 \times 10^{-6}$ \\
8 & $1.94362 \times 10^{-6}$ \\
9 & $4.13814 \times 10^{-7}$ \\
\hline
\end{tabular}

which is imposed with a set of Robin boundary conditions. The beam-type fractional BVP of order $3<\alpha \leq 4$ with separated boundary conditions and the product nonlinearity is provided in Example 4. Besides the obtained approximate solutions, we also provided the maximal errors $\left(\mathrm{ME}_{n}\right)$ and the error remainder functions $\left(\mathrm{ER}_{n}(x)\right)$ for each problem if possible. The results for all examples shown confirm that increasing the number of solution components (i.e., the value of $n$ ) reduces the errors in the numerical solutions. Furthermore, unlike the method of undetermined coefficients in the ADM, the Duan-Rach modified decomposition method does not require solving a system of nonlinear algebraic equations obtained from using the $n$-term approximation $\varphi_{n}(x)$ for the remaining unknown constants of integration, which are sometimes multiple roots or nonphysical roots. Hence, the method is very efficient and has provided very accurate approximate solutions when compared with the exact solutions (if any).

\section{Conflicts of Interest}

The authors declare that there are no conflicts of interest regarding the publication of this paper.

\section{References}

[1] S. Kumar, "A new fractional modeling arising in engineering sciences and its analytical approximate solution," Alexandria Engineering Journal, vol. 52, no. 4, pp. 813-819, 2013.
[2] C. Tricaud and Y. Q. Chen, "An approximate method for numerically solving fractional order optimal control problems of general form," Computers and Mathematics with Applications, vol. 59, no. 5, pp. 1644-1655, 2010.

[3] R. Magin, X. Feng, and D. Baleanu, "Solving the fractional order Bloch equation," Concepts in Magnetic Resonance Part A: Bridging Education and Research, vol. 34, no. 1, pp. 16-23, 2009.

[4] J. He, "Nonlinear oscillation with fractional derivative and its applications," in Proceedings of the International conference on vibrating engineering, pp. 288-291, 1998.

[5] I. Podlubny, Fractional Differential Equations, vol. 198 of Mathematics in Science and Engineering, Academic Press, San Diego, Calif, USA, 1999.

[6] C. Lederman, J.-M. Roquejoffre, and N. Wolanski, "Mathematical justification of a nonlinear integrodifferential equation for the propagation of spherical flames," Annali di Matematica Pura ed Applicata. Series IV, vol. 183, no. 2, pp. 173-239, 2004.

[7] A. Carvalho and C. M. Pinto, "A delay fractional order model for the co-infection of malaria and HIV/AIDS," International Journal of Dynamics and Control, vol. 5, no. 1, pp. 168-186, 2017.

[8] N. Ozalp and E. Demirci, "A fractional order SEIR model with vertical transmission," Mathematical and Computer Modelling, vol. 54, no. 1-2, pp. 1-6, 2011.

[9] G. Adomian, "Solution of physical problems by decomposition," Computers and Mathematics with Applications, vol. 27, no. 9-10, pp. 145-154, 1994.

[10] H. Jafari and V. Daftardar-Gejji, "Positive solutions of nonlinear fractional boundary value problems using Adomian decomposition method," Applied Mathematics and Computation, vol. 180, no. 2, pp. 700-706, 2006.

[11] J.-S. Duan, T. Chaolu, R. Rach, and L. Lu, “The Adomian decomposition method with convergence acceleration techniques for nonlinear fractional differential equations," Computers and Mathematics with Applications, vol. 66, no. 5, pp. 728-736, 2013.

[12] A.-M. Wazwaz, "Adomian decomposition method for a reliable treatment of the Bratu-type equations," Applied Mathematics and Computation, vol. 166, no. 3, pp. 652-663, 2005.

[13] S. Momani and N. Shawagfeh, "Decomposition method for solving fractional Riccati differential equations," Applied Mathematics and Computation, vol. 182, no. 2, pp. 1083-1092, 2006.

[14] J. S. Duan, R. Rach, D. Baleanu, and A. M. Wazwaz, "A review of the Adomian decomposition method and its applications to fractional differential equations," Communications in Fractional Calculus, vol. 3, no. 2, 2012.

[15] V. S. Ertürk, G. Zaman, and S. Momani, "A numeric-analytic method for approximating a giving up smoking model containing fractional derivatives," Computers and Mathematics with Applications, vol. 64, no. 10, pp. 3065-3074, 2012.

[16] I. Petras, "Modeling and numerical analysis of fractional-order bloch equations," Computers Mathematics with Applications, vol. 61, no. 2, pp. 341-356, 2011.

[17] U. Saeed and M. ur Rehman, "Haar wavelet operational matrix method for fractional oscillation equations," International Journal of Mathematics and Mathematical Sciences, Article ID 174819, Art. ID 174819, 8 pages, 2014.

[18] I. Hashim, M. S. M. Noorani, R. Ahmad, S. A. Bakar, E. S. Ismail, and A. M. Zakaria, "Accuracy of the adomian decomposition method applied to the Lorenz system," Chaos, Solitons \& Fractals, vol. 28, no. 5, pp. 1149-1158, 2006.

[19] N. H. Sweilam and M. M. Khader, "Approximate solutions to the nonlinear vibrations of multiwalled carbon nanotubes using 
Adomian decomposition method," Applied Mathematics and Computation, vol. 217, no. 2, pp. 495-505, 2010.

[20] M. M. Hosseini, "Adomian decomposition method with Chebyshev polynomials," Applied Mathematics and Computation, vol. 175, no. 2, pp. 1685-1693, 2006.

[21] Y. Liu, "Adomian decomposition method with orthogonal polynomials: Legendre polynomials," Mathematical and Computer Modelling, vol. 49, no. 5-6, pp. 1268-1273, 2009.

[22] W.-C. Tien and C.-K. Chen, "Adomian decomposition method by Legendre polynomials," Chaos, Solitons and Fractals, vol. 39, no. 5, pp. 2093-2101, 2009.

[23] P. V. Ramana and B. K. R. Prasad, "Modified Adomian Decomposition Method for Van der Pol equations," International Journal of Non-Linear Mechanics, vol. 65, pp. 121-132, 2014.

[24] M. Tatari and M. Dehghan, "The use of the Adomian decomposition method for solving multipoint boundary value problems," Physica Scripta, vol. 73, no. 6, article no. 023, pp. 672-676, 2006.

[25] W. Al-Hayani, "Adomian decomposition method with Green's function for sixth-order boundary value problems," Computers \& Mathematics with Applications, vol. 61, no. 6, pp. 1567-1575, 2011.

[26] J.-S. Duan and R. Rach, "A new modification of the Adomian decomposition method for solving boundary value problems for higher order nonlinear differential equations," Applied Mathematics and Computation, vol. 218, no. 8, pp. 4090-4118, 2011.

[27] J.-S. Duan, R. Rach, A.-M. Wazwaz, T. Chaolu, and Z. Wang, "A new modified Adomian decomposition method and its multistage form for solving nonlinear boundary value problems with Robin boundary conditions," Applied Mathematical Modelling. Simulation and Computation for Engineering and Environmental Systems, vol. 37, no. 20-21, pp. 8687-8708, 2013.

[28] Y. Lin, Y. Liu, and Z. Li, "Symbolic computation of analytic approximate solutions for nonlinear fractional differential equations," Computer Physics Communications, vol. 184, no. 1, pp. 130-141, 2013.

[29] O. Kelesoglu, "The solution of fourth order boundary value problem arising out of the beam-column theory using Adomian decomposition method," Mathematical Problems in Engineering, Article ID 649471, 6 pages, 2014.

[30] J.-S. Duan, R. Rach, and A.-M. Wazwaz, "Solution of the model of beam-type micro- and nano-scale electrostatic actuators by a new modified Adomian decomposition method for nonlinear boundary value problems," International Journal of Non-Linear Mechanics, vol. 49, pp. 159-169, 2013.

[31] B. Jang, "Two-point boundary value problems by the extended Adomian decomposition method," Journal of Computational and Applied Mathematics, vol. 219, no. 1, pp. 253-262, 2008.

[32] N. T. Shawagfeh, "Analytical approximate solutions for nonlinear fractional differential equations," Applied Mathematics and Computation, vol. 131, no. 2-3, pp. 517-529, 2002.

[33] S. G. Samko, A. A. Kilbas, and O. I. Marichev, Fractional Integrals and Derivatives, Theory and Applications, Gordon and Breach, Yverdon, Switzerland, 1993.

[34] A. A. Kilbas, H. M. Srivastava, and J. J. Trujillo, "Preface," NorthHolland Mathematics Studies, vol. 204, no. C, pp. vii-x, 2006.

[35] S. Das, Functional Fractional Calculus, Springer, New Delhi, India, 2011.

[36] G. Adomian, "A review of the decomposition method and some recent results for nonlinear equations," Computers \& Mathematics with Applications, vol. 21, no. 5, pp. 101-127, 1991.
[37] G. Adomian and R. Rach, "Inversion of nonlinear stochastic operators," Journal of Mathematical Analysis and Applications, vol. 91, no. 1, pp. 39-46, 1983.

[38] G. Adomian, "A review of the decomposition method and some recent results for nonlinear equations," Mathematical and Computer Modelling, vol. 13, no. 7, pp. 17-43, 1990.

[39] A.-M. Wazwaz, "A reliable modification of Adomian decomposition method," Applied Mathematics and Computation, vol. 102, no. 1, pp. 77-86, 1999.

[40] J.-S. Duan, "Recurrence triangle for Adomian polynomials," Applied Mathematics and Computation, vol. 216, no. 4, pp. 12351241, 2010.

[41] J.-S. Duan and R. Rach, "On the domain of convergence of the Adomian series solution," Applied Mathematics and Computation, vol. 66, no. 5, pp. 728-736, 2013.

[42] J. S. Duan, R. Rach, and Z. Wang, "On the effective region of convergence of the decomposition series solution," Journal of Algorithms Computational Technology, vol. 7, no. 2, pp. 287-247, 2012.

[43] A. Wazwaz, "Approximate solutions to boundary value problems of higher order by the modified decomposition method," Computers \& Mathematics with Applications, vol. 40, no. 6-7, pp. 679-691, 2000.

[44] A. Wazwaz, Partial Differential Equations and Solitary Waves Theory, Higher Education Press, Beijing, China; Springer, Berlin, Germany, 2009.

[45] U. M. Ascher, R. M. Mattheij, and R. D. Russell, Numerical Solution of Boundary Value Problems for Ordinary Differential Equations, SIAM, Philadelphia, Pa, USA, 1995.

[46] J. P. Boyd, "Chebyshev polynomial expansions for simultaneous approximation of two branches of a function with application to the one-dimensional Bratu equation," Applied Mathematics and Computation, vol. 143, no. 2-3, pp. 189-200, 2003.

[47] Y.-T. Yang, S.-K. Chien, and C.-K. Chen, "A double decomposition method for solving the periodic base temperature in convective longitudinal fins," Energy Conversion and Management, vol. 49, no. 10, pp. 2910-2916, 2008. 


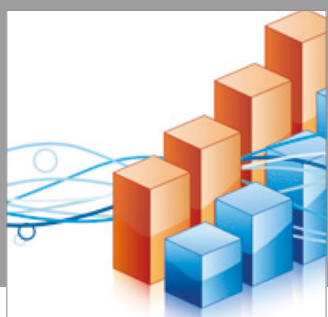

Advances in

Operations Research

vatersals

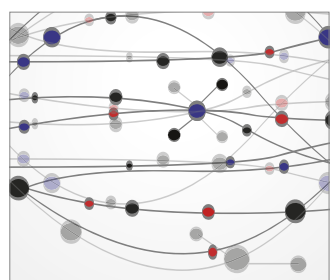

\section{The Scientific} World Journal
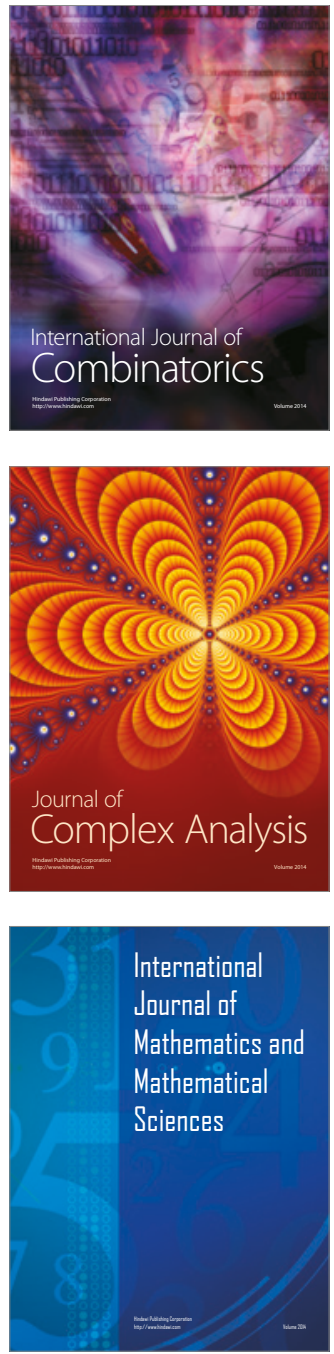
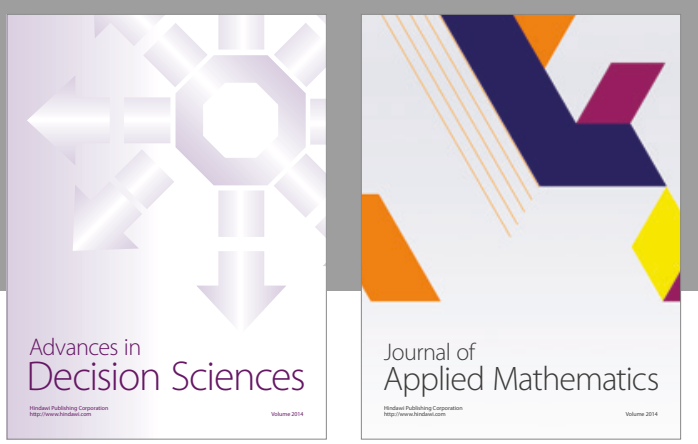

Algebra

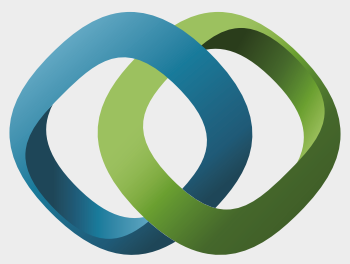

\section{Hindawi}

Submit your manuscripts at

https://www.hindawi.com
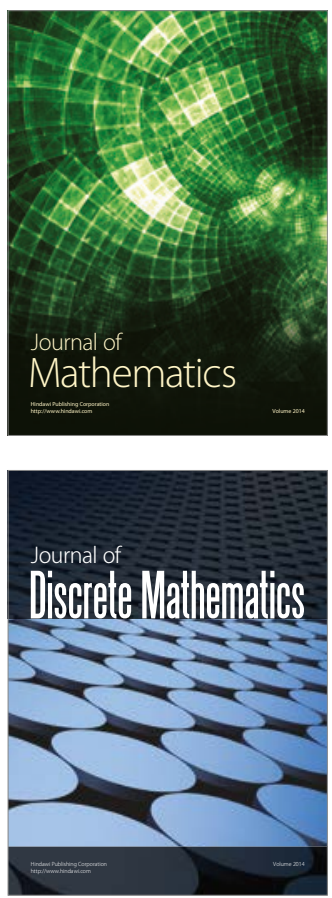

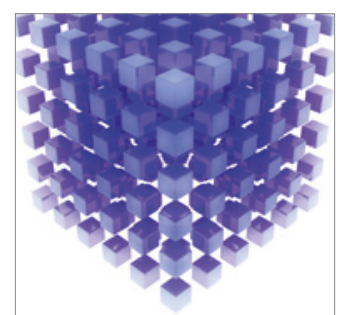

Mathematical Problems in Engineering
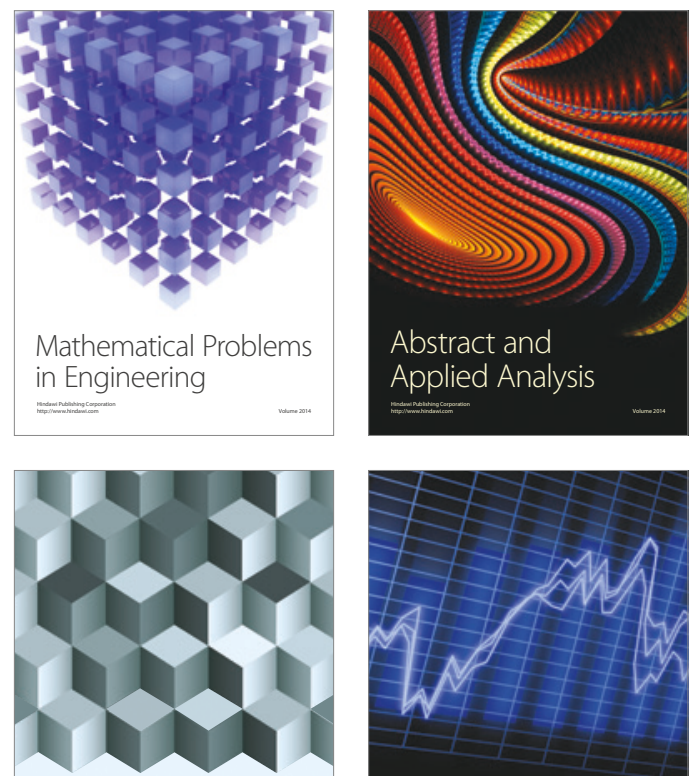

Journal of

Function Spaces

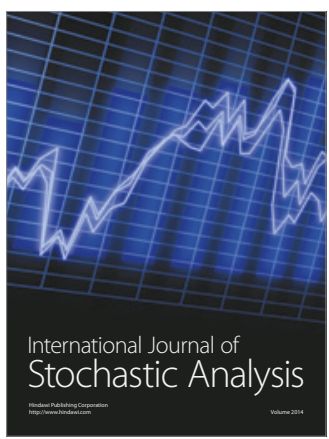

Probability and Statistics
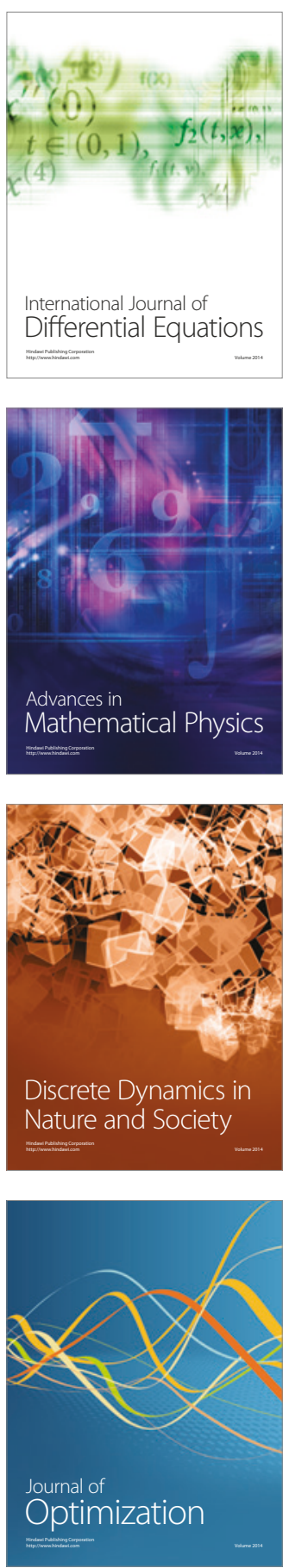